\title{
A First Passage Based Model for Probabilistic Fracture of Polycrystalline Silicon MEMS Structures
}

\author{
Zhifeng $\mathrm{Xu}^{1}$ and Jia-Liang $\mathrm{Le}^{2}$
}

\begin{abstract}
Experiments have shown that the failure loads of Microelectronicalmechanical Systems (MEMS) devices are subjected to a considerable level of variability, which is believed to be caused by the random material strength and the geometry-induced random stress field. Understanding the strength statistics of MEMS devices is of paramount importance for the device design guarding against a tolerable failure risk. In this study, we develop a continuum-based probabilistic model for polycrystalline silicon (poly-Si) MEMS structures within the framework of first passage analysis. The failure of poly-Si MEMS structures is considered to be triggered by fracture initiation from the sidewalls, which is described by a nonlocal failure criterion. The model takes into account an autocorrelated random field of material tensile strength. The random nonlocal stress field is obtained by stochastic finite element simulations based on the information of the uncertainties of the sidewall geometry. The model is formulated within the context of both stationary and non-stationary stochastic processes for MEMS structures of various geometries and under different loading configurations. It is shown that the model agrees well with the experimentally measured strength distributions of uniaxial tensile poly-Si MEMS specimens of different gauge lengths. The model is further used to predict the strength distribution of poly-Si MEMS beams under three-point bending, and the result is compared with the Monte Carlo simulation. The present model predicts a strong size effect on the mean structural strength, which consists of three power-law asymptotes in the small, intermediate, and large-size regimes. By matching these three asymptotes, an approximate size effect equation is proposed. The present model is shown to be a generalization of the classical weakest-link statistical model, and it provides a physical interpretation of the material length scale used in the weakest-link model.
\end{abstract}

Keywords: Asymptotic analysis, Weakest-link model, Size effect, Structural reliability, Stochastic process

\section{Introduction}

Microelectronicalmechanical Systems (MEMS) devices offer a range of applications in many areas such as medical technology, transportation systems, energy conversion and biochemical threat detection. In some of these applications, MEMS devices are required to operate at high mechanical power densities and/or a large deformation level, for which the strength limit becomes an important design consideration. Over the past decade, there has been a continuing interest in understanding the probability distribution of the strength of MEMS devices. Experiments have shown that, for MEMS devices made of various materials such as polycrystalline silicon (poly-Si), single crystal silicon, silicon carbide, ultrananocrystalline diamond and hydrogen-free tetrahedral amorphous carbon, the measured structural strength exhibits a considerable level of variability $[11,8,14]$. Such variability is related to the random geometry of the sidewall grooves $[8,7,20]$, which is an unavoidable outcome of the fabrication process.

Experimental histogram testing provides the most direct means of investigating the strength distribution of MEMS structures. However, in many cases experimental investigation alone is insufficient for the purpose of reliability-based design of MEMS devices due to two reasons: 1) The design of MEMS devices is often required to ensure a low failure risk (e.g. a failure probability of $10^{-4}$ ). However, conventional histogram testing is unable to probe the tail distribution unless an excessively large number of specimens are used, which is cost prohibitive. 2) Histogram testing is usually limited to

\footnotetext{
${ }^{1}$ Graduate Research Assistant, Department of Civil, Environmental, and Geo- Engineering, University of Minnesota, Minneapolis, Minnesota.

${ }^{2}$ Associate Professor, Department of Civil, Environmental, and Geo- Engineering, University of Minnesota, Minneapolis, Minnesota. Email: jle@umn.edu. Corresponding author.
} 
some simple specimen geometries and loading configurations, which are different from the actual applications. Therefore, analytical modeling becomes an indispensable tool for reliability-based analysis and design of MEMS structures.

The existing analytical modeling of strength statistics of MEMS structures is largely based on the weakest-link models. The most widely used model of such a kind is the two-parameter Weibull distribution, which is one of the three extreme value distributions $[13,17,25]$. The statistical parameters of the Weibull distribution can be determined by its first and second moments. Furthermore, by using the Weibull statistical model, one can easily extrapolate the strength distributions of specimens across different geometries and sizes. It can also be shown that the two-parameter Weibull distribution leads to a power-law size effect on the mean strength, which is often referred to as the Weibull size effect $[15,6]$.

The main underlying assumption of the Weibull statistics is that the structure can be statistically modeled as an infinite chain of independent elements. However, it appears that, for many MEMS structures, the sidewall length is not sufficiently long to grant the validity of this assumption. This is manifested by the fact that, for MEMS structures made of various materials, the experimentally measured strength histograms consistently deviate from the two-parameter Weibull distribution [11, $8,14,23]$. In view of this deviation, the three-parameter Weibull distribution has been suggested as an empirical model to improve the fitting of strength histograms. Recent studies investigated the strength distributions of two sets of poly-Si MEMS specimens, which have different gauge lengths [23]. This experimental result allowed a more complete model validation. In a recent analysis of this experiment [20], the measured strength statistics of specimens of one gauge length was used to calibrate the analytical model, and the model was then used to predict the strength statistics of specimens of the other gauge length. It was shown that, despite the limited size range of the specimens, the predictability of the three-parameter Weibull distribution strongly depends on which set of specimens was used for calibration.

To relax the assumption of the classical Weibull statistics, a finite weakest-link model was recently proposed to account for the finite-size geometry of MEMS structures [20]. The model also considers the random stress field induced by the random sidewall geometry. The comparison with the aforementioned test by Reedy Jr. et al. [23] showed that the model agrees well with the strength distribution of poly-Si MEMS specimens of two sizes, and the values of the model parameters mildly depend on the choice of the set of specimens used for calibration. This model is anchored by three main simplified assumptions: 1) the surface grooves of the sidewall are non-interacting, 2) there are a fixed number of surface grooves for a given length of the sidewall, and 3) the material strength of the region of each surface groove is statistically independent. These assumptions imply that we can treat the failure statistics of each surface groove to be statistically independent, which is an essential assumption of the weakest-link model. However, the actual fabrication of MEMS specimens inevitably introduces randomly spaced surface grooves, and consequently the number of grooves for specimens of a given sidewall length must be random. The random spacing of surface grooves also causes potential interactions between the neartip stress fields. For closely spaced surface grooves, it is also necessary to consider the autocorrelation feature of the random strength field, which cannot be directly handled by the weakest-link models [26]. Therefore, a more general probabilistic model is needed.

In this study, we develop a new probabilistic model for the failure of MEMS structures based on the first passage analysis. The main feature of the model is that it takes into account the statistical interdependence of the failure risks of material points, which leads to a more physical description of the failure of MEMS structures. This paper is planned as follows: Section 2 presents the formulation of this model based on the principle of stationary stochastic process; Section 3 extends the formulation to the case where the failure statistics is calculated by using the concept of non-stationary stochastic process; Section 4 compares the model with the measured strength distributions of uniaxial tensile poly-Si MEMS specimens of two gauge lengths; Section 5 applies the model to poly-Si MEMS specimens under three-point bending; Section 6 presents the predicted size effect on the probability distribution 
of MEMS specimens including its corresponding asymptotic behaviors, Section 7 discusses the size effect on the mean structural strength and its approximation using asymptotic analysis; and Section 8 presents the relation between the present model and the conventional weakest-link statistical model of structural failure.

\section{Model Formulation Based on Stationary Stochastic Process}

We first consider uniaxial tensile MEMS specimens subjected to a far-field stress $\sigma_{0}$. The sidewalls of the specimen consist of a series of randomly spaced surface grooves induced by the fabrication process (Fig. 1). These grooves can be modeled as V-notches for the purpose of stress analysis $[22,23,20]$. Since the tensile specimen is of positive geometry (i.e. the stress intensity factor increases with the crack length) [5,21], we may consider that the specimen reaches its peak load capacity at fracture initiation. Due to the randomness of material strength and stress field, the location of fracture initiation point is intrinsically random.

We note that, for typical MEMS specimens, the distance between the two opposite sidewalls is much larger than the size of the surface grooves. Therefore, the surface grooves of two sidewalls would not interact with each other. To formulate the failure criterion, it suffices to consider a specimen consisting of only one sidewall with random spaced V-notches. We first determine the maximum elastic stress $\sigma_{x x}$ in $y$-direction for any given coordinate $x$, and then compute an average stress of its neighborhood of size $l_{y}$, i.e.

$$
\sigma(x)=l_{y}^{-1} \int_{l_{y}} \sigma_{x x}(x, y) \mathrm{d} y
$$

Due to the presence of $\mathrm{V}$-notches, the maximum elastic stress $\sigma_{x x}$ occurs very close to the sidewall (i.e. either at the notch tip or close to the line connecting two adjacent notches). Therefore, it is reasonable to consider that the failure of these MEMS specimens is induced by fracture initiation from the sidewall. Eq. 1 can be considered as a simplified version of the nonlocal averaging procedure, which takes into account the finite length of the fracture process zone (FPZ). $l_{y}$ can be taken approximately as the length of the FPZ. It is noted that this averaging process is necessary for formulating the strength-based failure criterion since the material points at the $\mathrm{V}$-notch tips would experience a singular stress. To consider the width of the FPZ, we further introduce a nonlocal averaging for stress $\sigma(x)$ in $x$-direction:

$$
\bar{\sigma}(x)=\int_{0}^{\infty} \alpha\left(\left|x^{\prime}-x\right|\right) \sigma\left(x^{\prime}\right) \mathrm{d} x^{\prime}
$$

where $\alpha(r)=\left[1-\left(r / \rho_{0} l_{x}\right)^{2}\right]^{2}\left(\rho_{0}=15 / 16\right)$. The nonlocal stress $\bar{\sigma}(x)$ can be considered as an average stress measure of the FPZ. We may consider that fracture initiates (or, equivalently, the FPZ is fully formed) once the nonlocal stress $\bar{\sigma}(x)$ reaches the material tensile strength $f_{t}$, and at that instant the overall specimen attains its peak load capacity. This nonlocal failure criterion clearly contains the information of FPZ size, and it allows us to formulate a continuum probabilistic failure model, which naturally leads to the first passage analysis.

Since the aforementioned stress analysis is purely based on linear elasticity, we can write $\bar{\sigma}(x)=$ $\sigma_{0} Z(x)$, where $Z(x)=$ dimensionless stress field. It is evident that $Z(x)$ can be viewed as a random field since the stress field would depend on the random geometry of the sidewall. Meanwhile, the material tensile strength $f_{t}$ is also subjected to a certain level of spatial variability. Denote the strength of the specimen as $\sigma_{m}$ (i.e. the maximum value of $\sigma_{0}$ ). According to the aforementioned failure criterion, we can express the probability distribution of $\sigma_{m}$ (i.e. the probability that $\sigma_{m}$ does not exceed a prescribed value $\sigma_{N}$ ) for a tensile specimen of sidewall length $L$ as

$$
F\left(\sigma_{N}\right)=\operatorname{Pr}\left(\sigma_{m} \leq \sigma_{N}\right)=1-\operatorname{Pr}\left[\sigma_{N} Z(x) \leq f_{t}(x), \forall x \in L\right]
$$

The randomness of the geometry of surface grooves does not have a spatial bias along the sidewall. Therefore, it is clear that, for uniaxial tension specimens, we can consider both $Z(x)$ and $f_{t}(x)$ as 
stationary stochastic processes. To compute the probability in Eq. 3, it is convenient to decompose the random field into its mean value and a random fluctuation component:

$$
\begin{gathered}
f_{t}(x)=\mu_{f_{t}}+f_{t 0}(x) \\
Z(x)=\mu_{z}+Z_{0}(x)
\end{gathered}
$$

where $\mu_{f_{t}}$ and $\mu_{z}$ are the mean values of $f_{t}(x)$ and $Z(x)$, respectively. The expectations of $f_{t 0}(x)$ and $Z_{0}(x)$ are equal to 0 . The random field $f_{t}(x)$ can be simulated by using a known probability distribution function and a correlation function, whereas the process $\left.Z_{(} x\right)$ can be obtained by stochastic simulation of the stress field. The autovariance-ergodicity of the zero-mean process $Z_{0}(x)$ can be examined by the following condition:

$\Psi(\epsilon)=\lim _{l \rightarrow \infty} \frac{2}{l} \int_{0}^{l}\left(1-\frac{\xi}{l}\right)\left\{\mathrm{E}\left\{Z_{0}(x) Z_{0}(x+\epsilon) Z_{0}(x+\xi) Z_{0}(x+\epsilon+\xi)\right\}-\left\{\mathrm{E}\left[Z_{0}(x) Z_{0}(x+\epsilon)\right]\right\}^{2}\right\} \mathrm{d} \xi=0 \quad \forall \epsilon \in l$

where $\mathrm{E}(\cdot)$ denotes the expectation. Note that, if we choose $\epsilon=0$, Eq. 5 reduces to the requirement for a mean-ergodic process.

Substituting Eqs. 4a and 4b into Eq. 3, we can write the cumulative distribution function (cdf) of the tensile strength of the specimen as

$$
F\left(\sigma_{N}\right)=1-\operatorname{Pr}\left[\eta_{0}(x) \leq \lambda, \forall x \in L\right]
$$

where $\eta_{0}(x)=\sigma_{N} Z_{0}(x)-f_{t 0}(x)$ and $\lambda=\mu_{f_{t}}-\sigma_{N} \mu_{z}$. Following the foregoing analysis, it is clear that $\eta_{0}(x)$ is a zero mean stationary process, which depends on $\sigma_{N}$. The probability term $\operatorname{Pr}\left[\eta_{0}(x) \leq \lambda, \forall x \in L\right]$ refers to the probability that the value of the stochastic process $\eta_{0}(x)$ never crosses the barrier $\lambda$ for all $x \in[0, L]$ (Fig. 2). This probability can be computed by using the first passage analysis $[24,25]$ :

$$
\operatorname{Pr}\left[\eta_{0}(x) \leq \lambda, \forall x \in L\right]=F_{\eta_{0}}(\lambda) \exp \left[-\frac{\mu_{\lambda}}{F_{\eta_{0}}(\lambda)} L\right]
$$

where $F_{\eta_{0}}=$ cdf of $\eta_{0}$, and $\mu_{\lambda}=$ crossing rate. Eq. 7 essentially employs an exponential decay behavior of the reliability function. $F_{\eta_{0}}(\lambda)$ takes into account the non-crossing probability at the starting point. For uniaxial tension specimens, the choice of this starting point does not matter. The term $\mu_{\lambda} / F_{\eta_{0}}(\lambda)$ accounts for the finite distance of crossing of the barrier [25].

Since the random fields $Z_{0}(x)$ and $f_{t 0}(x)$ are mutually independent, the probability density function (pdf) of $\eta_{0}$ can be calculated from the pdfs of $Z_{0}$ and $f_{t 0}$ as

$$
f_{\eta_{0}}(\eta)=\int_{-\infty}^{\infty} \sigma_{N}^{-1} f_{Z_{0}}\left(z / \sigma_{N}\right) f_{f_{t 0}}(z-\eta) \mathrm{d} z
$$

where $f_{Z_{0}}$ and $f_{t 0}$ denote the pdfs of $Z_{0}$ and $f_{t 0}$, respectively. The crossing rate $\mu_{\lambda}$ essentially represents the mean rate at which the stochastic process $\eta_{0}(x)$ crosses over the barrier $\lambda$, which is governed by the expectation of the positive gradient of $\eta_{0}(x)$ at the barrier, i.e.:

$$
\begin{aligned}
\mu_{\lambda} & =\mathrm{E}\left[\dot{\eta}_{0}(x)^{+} \mid \eta_{0}(x)=\lambda\right] \\
& =\int_{0}^{\infty} \zeta f_{\eta_{0} \dot{\eta}_{0}}(\lambda, \zeta) \mathrm{d} \zeta
\end{aligned}
$$

where $\dot{\eta}_{0}(x)=\mathrm{d} \eta_{0}(x) / \mathrm{d} x$, the subscript " + " denotes the positive gradient, and $f_{\eta_{0} \dot{\eta}_{0}}=$ joint pdf of $\eta_{0}$ and $\dot{\eta}_{0}$.

The probability distribution of the stochastic process $\eta_{0}(x)$ is generally non-Gaussian, and therefore there is no guarantee that $\eta_{0}(x)$ and $\dot{\eta}_{0}(x)$ would be statistically independent. The joint pdf $f_{\eta_{0}} \dot{\eta}_{0}$ is 
usually unavailable, which makes it difficult to calculate the mean crossing rate $\mu_{\lambda}$ for a non-Gaussian stochastic process. To circumvent this difficulty, it has been proposed to transform the process to a standard Gaussian process, from which the actual mean crossing rate can be well estimated [16]. The transformed standard Gaussian process $\psi_{0}(x)$ can be related to the original stochastic process $\eta_{0}(x)$ as

$$
\psi_{0}(x)=\Phi^{-1}\left\{F_{\eta_{0}}\left[\eta_{0}(x)\right]\right\}
$$

where $\Phi(z)=$ standard Gaussian cdf. For the standard Gaussian process, it can be shown that the random process of the original variable is statistically independent of the random process representing the gradient of the variable [16]. Therefore, Eq. 10 can be rewritten as

$$
\mu_{\lambda} \approx \phi\left(\lambda_{G}\right) \int_{0}^{\infty} \psi f_{\dot{\psi}_{0}}(\psi) \mathrm{d} \psi=\frac{\delta_{\dot{\psi}_{0}}}{\sqrt{2 \pi}} \phi\left(\lambda_{G}\right)
$$

where $\phi(z)=$ standard Gaussian pdf, $\dot{\psi}_{0}(x)=\mathrm{d} \psi_{0}(x) / \mathrm{d} x$, and $\delta_{\dot{\psi}_{0}}=$ standard deviation of $\dot{\psi}_{0}(x)$. Based on Eq. 11, the equivalent crossing barrier $\lambda_{G}$ of the transformed Gaussian field is related to the original crossing barrier $\lambda$ as $\lambda_{G}=\Phi^{-1}\left[F_{\eta_{0}}(\lambda)\right]$. The standard deviation $\delta_{\dot{\psi}_{0}}$ can directly be calculated by differentiating the random field $\psi_{0}(x)$. A better approach to determine $\delta_{\dot{\psi}_{0}}$ is through the power spectral density of $\eta_{0}(x)$, which avoids the differentiation of the random field, i.e.

$$
\delta_{\dot{\psi}_{0}}=\left[\int_{-\infty}^{\infty} \omega^{2} S_{\psi_{0}}(\omega) \mathrm{d} \omega\right]^{1 / 2}
$$

where the power spectral density $S_{\psi_{0}}(\omega)$ can be expressed as

$$
S_{\psi_{0}}(\omega)=\lim _{l \rightarrow \infty} \mathrm{E}\left[\frac{1}{2 \pi l}\left|\int_{0}^{l} \psi_{0}(x) e^{-i \omega x} \mathrm{~d} x\right|^{2}\right]
$$

Using Eqs. 6-8, 12 and 13, we can compute the strength distribution of a uniaxial tensile specimen of any sidewall length $L$. It is worthwhile to briefly comment on the asymptotic behaviors of this strength cdf based on Eqs. 6 and 7. As the specimen size approaches to 0, the model essentially implies that the strength cdf is governed by the failure probability of a material point. We will show in Section 7 that this feature is essential for constructing a meaningful small-size asymptote of the size effect curve of the mean structural strength. At the large-size limit $(L \rightarrow \infty)$, it is expected that the failure statistics would be governed by small values of $\sigma_{N}$. In other words, the value of the crossing barrier would be high, which implies $F_{\eta_{0}}(\lambda) \approx 1$. Therefore, Eq. 6 becomes

$$
F\left(\sigma_{N}\right)=1-\exp \left[-\mu_{\lambda}\left(\sigma_{N}\right) L\right]
$$

It is noted that Eq. 15 resembles the form of the Weibull weakest-link model $[27,28]$, which has widely been used for characterization of the statistics of material strength. As will be discussed later, indeed the present model can be translated to the weakest-link model, which guarantees that the large-size asymptotic behavior of this model is consistent with the classical theory of the extreme value statistics $[13,17]$.

\section{Failure Statistics Modeled by Non-Stationary Stochastic Process}

The foregoing analysis is strictly anchored by the assumption that $\eta_{0}(x)$ can be considered as a stationary stochastic process. As discussed earlier, such an assumption is valid for uniaxial tension specimens. However, for specimens of other geometries or under different loading configurations, such as three-point bend specimens, the dimensionless stress field $Z(x)$ could lose its stationarity, and therefore $\eta_{0}(x)$ has to be considered as a non-stationary stochastic process. This section extends the model formulated in Section 2 to the non-stationary case. It should be noted that we still limit our 
attention to the scenario where the failure always initiates from the sidewall. This implies that $Z(x)$ is a $1 \mathrm{D}$ stochastic process.

Consider that the stress field $Z(x)$ is represented by a non-stationary process whereas the strength field $f_{t}(x)$ remains a stationary process. Denote the nominal strength of the specimen as $\sigma_{m}=$ $P_{\max } / b D$, where $P_{\max }=$ maximum load capacity, $D=$ characteristic size of the specimen, $b=$ width of the specimen in the transverse direction. Within the framework of linear elasticity, the stress field at the peak load can be expressed as $\sigma(x)=\sigma_{m} Z(x)$. The random stress field $Z(x)$ can further be written as

$$
Z(x)=\mu_{z}(x)\left[1+Z_{n}^{0}(x)\right]
$$

where the mean value $\mu_{z}(x)$ varies spatially and $Z_{n}^{0}(x)$ should generally be considered as a zero-mean non-stationary process. The probability distribution of nominal strength $\sigma_{m}$ can be expressed as

$$
\begin{aligned}
F\left(\sigma_{N}\right) & =1-\operatorname{Pr}\left[\eta_{n}(x) \leq \lambda(x), \forall x \in L\right] \\
\text { where: } \lambda(x) & =\mu_{f_{t}}-\sigma_{N} \mu_{z}(x) \\
\eta_{n}(x) & =\sigma_{N} \mu_{z}(x) Z_{n}^{0}(x)-f_{t 0}(x)
\end{aligned}
$$

By introducing the non-stationary process, we expect that the mean crossing rate of the failure process is not uniform along the sidewall. In such a case, Eq. 7 can be generalized as

$$
F\left(\sigma_{N}\right)=1-F_{\eta_{n}^{0}}\left(\lambda_{0}\right) \exp \left\{-\int_{0}^{L} \frac{\mu_{\lambda}(x)}{F_{\eta_{n \mid x}}[\lambda(x)]} \mathrm{d} x\right\}
$$

where $F_{\eta_{n \mid x}}=\mathrm{cdf}$ of $\eta_{n}$ at coordinate $x, \mu_{\lambda}(x)=$ mean crossing rate at coordinate $x$ and $F_{\eta_{n}^{0}}=$ cdf of $\eta_{n}$ at point $x_{0}$ where $\mu_{z}(x)$ attains its maximum value, and $\lambda_{0}$ is the crossing barrier at point $x_{0}$. For a given specimen geometry, we expect that the strength cdf of small-size specimens will be governed by the failure statistics at the point of the maximum mean stress. This small-size asymptotic behavior is reflected in Eq. 20: $F\left(\sigma_{N}\right)=1-F_{\eta_{n}^{0}}\left(\lambda_{0}\right)$ as $L \rightarrow 0$.

In principle, the mean crossing rate for a non-stationary process can also be estimated by using the concept of transformed Gaussian variable, which is similar to the previous analysis of stationary process. By using Eqs. 10 and 12, we can express $\mu_{\lambda}(x)$ as

$$
\begin{aligned}
\mu_{\lambda}(x) & =\int_{0}^{\infty} \zeta f_{\eta_{n \mid x} \dot{\eta}_{n \mid x}}[\lambda(x), \zeta, x] \mathrm{d} \zeta \\
& \approx \frac{\delta_{\dot{\psi}}(x)}{\sqrt{2 \pi}} \phi\left[\lambda_{G}(x)\right]
\end{aligned}
$$

where $f_{\eta_{n \mid x} \dot{\eta}_{n \mid x}}=$ joint pdf of $\eta_{n}$ and $\dot{\eta}_{n}$ at coordinate $x, \delta_{\dot{\psi}}(x)=$ standard deviation of the gradient of the transformed Gaussian variable at coordinate $x$, and $\lambda_{G}(x)=\Phi^{-1}\left\{F_{\eta_{n \mid x}}[\lambda(x)]\right\}$, which corresponds to the crossing barrier of the transformed Gaussian variable. The essential step of the aforementioned method is the transformation of the non-stationary process to an equivalent Gaussian process. This transformation is a challenging task, which has attracted considerable attention over the past decade $[9,12,19]$. Furthermore, it is noted that Eq. 20 is computationally demanding since it involves the evaluation of the cdf of $Z_{n}^{0}(x)$ and the standard deviation of the gradient of the transformed Gaussian variable $\dot{\psi}$ at every point. Therefore, it is desirable to introduce some approximations in order to simplify the analysis.

The most straightforward simplification is to approximate the underlying process $Z(x)$ by a uniformly modulated non-stationary process. As will be shown in Section 5, this is a reasonable approximation for MEMS specimens under flexural loading, which is a common loading configuration in the actual applications. With such an approximation, the process $Z_{n}^{0}(x)$ in Eq. 16 can be treated as a stationary stochastic process. The stationarity of $Z_{n}^{0}(x)$ can be examined by calculating the spatial 
variability of its covariance function. Furthermore, the ergodicity of $Z_{n}^{0}(x)$ can be checked by using Eq. 5. By considering $Z_{n}^{0}(x)$ as a stationary process, the cdf of $\eta_{n}$ at any point $x$ can be expressed as

$$
F_{\eta_{n}}(\eta)=\frac{1}{\sigma_{N} \mu_{z}(x)} \int_{-\infty}^{\infty} \int_{-\infty}^{\infty} f_{Z_{n}^{0}}\left[\zeta / \sigma_{N} \mu_{z}(x)\right] f_{f_{t 0}}\left(\zeta-\eta^{\prime}\right) \mathrm{d} \zeta \mathrm{d} \eta^{\prime}
$$

It is clear that Eq. 23 requires only the cdf of the stationary process $Z_{n}^{0}(x)$. For the calculation of Eq. 22 we just need to transform two stationary processes $Z_{n}^{0}(x), f_{t}(x)$ to the equivalent Gaussian processes, which is far more efficient than the transformation of the non-stationary process. These simplifications greatly reduce the computation effort for dealing with Eq. 20.

\section{Experimental Validation for Uniaxial Tension Specimens}

We now test the present model against the recent experimental results of the strength distributions of uniaxial tensile poly-Si specimens [23]. This experiment involved specimens of two gauge lengths $\left(L_{g}=20\right.$ and $\left.70 \mu \mathrm{m}\right)$. All specimens have a nominal width of $2 \mu \mathrm{m}$, which is much larger than the typical depth of a surface groove. In the test, specimens of $20 \mu \mathrm{m}$ gauge length were tested by using a recently developed slack-chain tester. This test apparatus is able to accommodate a large number of specimens in a chain loaded by a custom-built probe station. The main advantage of the slack-chain tester is that it allows simultaneous testing of a large number of replicates, which is ideal for histogram testing. Specimens of $70 \mu \mathrm{m}$ gauge length were tested by using an on-chip tester with a chevron thermal actuator. The details of these experimental set-ups were described in [18, 23].

To use the proposed model to analyze these experiments, we first determine the process $Z(x)$ by performing the stochastic simulation of the stress field. The simulation involves a finite element analysis of a uniaxial specimen of length of $1 \mathrm{~mm}$ and width of $2 \mu \mathrm{m}$ under a unit far-field stress $\left(\sigma_{0}=1\right)$. One sidewall of the specimen consists of a series of randomly spaced space grooves whereas the other sidewall is perfectly smooth. In the present analysis, the poly-Si material is modeled as an isotropic material with a Young Modulus $E=156 \mathrm{GPa}$ and a Poisson ratio $\mu=0.22$ [23], and the sidewall grooves are modeled as $\mathrm{V}$-notches. Based on some reported measurements of the sidewall geometry [23], the randomness of the sidewall geometry of the specimen is characterized by the following random variables (Fig. 1): 1) the angle $\theta$ of the $\mathrm{V}$-notches is assumed to follow a uniform distribution bounded between $20^{\circ}$ and $140^{\circ}, 2$ ) the depth $a$ of the $\mathrm{V}$-notches is described by the Type III extreme value distribution with an upper bound of $62 \mathrm{~nm}$, and 3) the spacing $s$ of the V-notches follows a uniform distribution bounded between $50 \mathrm{~nm}$ and $750 \mathrm{~nm}$, i.e.:

$$
\begin{array}{ll}
F_{\theta}(\theta)=\frac{\theta-20}{120} & \left(20^{\circ} \leq \theta \leq 140^{\circ}\right) \\
F_{a}(a)=\exp \left[-\left(\frac{62-a}{28}\right)^{6.5}\right] & (0 \leq a \leq 62 \mathrm{~nm}) \\
F_{s}(s)=\frac{s-50}{800} & (50 \mathrm{~nm} \leq s \leq 750 \mathrm{~nm})
\end{array}
$$

where $F_{\theta}(\theta), F_{a}(a)$ and $F_{s}(s)$ denote the cdfs of notch angle, notch depth, and notch spacing, respectively. In principle, the Type III extreme distribution could allow negative values of $a$, which is physically inadmissible in the present context. However, it is noted that the chance of sampling a negative value of $a$ is negligibly small, i.e. $F_{a}(0)=6.7 \times 10^{-77}$.

Based on these distribution functions, we generate a set of random V-notches over the entire specimen length. The simulated elastic stress field is then used to compute the stochastic process $Z(x)$ by using Eqs. 1 and 2. It is clear that the averaging length scales $\left(l_{x}\right.$ and $l_{y}$ in Eqs. 1 and 2$)$ are related to the FPZ size. Here we choose $l_{x}$ and $l_{y}$ to be $5 \mathrm{~nm}$, which is approximately on the order of the FPZ size of silicon $[29,23,20]$. It is clear that, to calculate the nonlocal stress, the finite element 
simulation needs to use fine meshes, whose size is on the order of nanometers. Though continuumbased finite element analysis has been used for stress analysis of surface grooves of poly-Si MEMS structures [23], the applicability of continuum mechanics at such a scale could be debatable. However, the essential point of the present model is to use an elastic stress-based nonlocal failure criterion to evaluate the failure risk of the specimen, in which the nonlocal stress averaging essentially accounts for the existence of the FPZ. It should be mentioned that different choices of nonlocal averaging functions would lead to different calibration results of the model parameters, but the qualitative behavior of the present model would remain the same.

In this study, we assume some simple nonlocal averaging functions and length scales $\left(l_{x}\right.$ and $\left.l_{y}\right)$, which as will be shown later yields a reasonable value of the mean strength of silicon. A physical approach to determine these continuum-based nonlocal averaging functions is to use atomistic simulations of the fracture behavior of the near-tip region (i.e. the behavior of FPZ).

Fig. 3a presents the simulated cdf of the process $Z(x)$, from which we can extract the underlying zero-mean process $Z_{0}(x)$. Fig. 3b shows that the normalized value of $\Psi(\epsilon)$ of Eq. 5 is approximately equal to zero indicating the ergodicity of $Z_{0}(x)$. It is also noted that $Z_{0}(x)$ has a clear autocorrelation feature (Fig. 3c). The autocorrelation length of $Z_{0}(x)$ (or $Z(x)$ ) is about $100 \mathrm{~nm}$, which reflects the potential interaction of the stress fields of the $\mathrm{V}$-notches governed by the random notch spacing.

Compared to the stochastic stress field $Z(x)$, the generation of random strength field $f_{t}(x)$ is relatively straightforward. The underlying cdf of $f_{t}(x)$ is considered to follow a Gauss-Weibull grafted distribution, which was recently derived for a single material element made of brittle or quasibrittle materials by using atomistic fracture mechanics and a multiscale statistical model [5, 4, 21]. The distribution function can be written as

$$
\begin{gathered}
F_{f_{t}}(\sigma)=1-\exp \left[-\left(\sigma / s_{0}\right)^{m}\right] \quad\left(\sigma \leq \sigma_{g r}\right) \\
F_{f_{t}}(\sigma)=P_{g r}+\frac{r_{f}}{\sqrt{2 \pi} \delta_{G}} \int_{\sigma_{g r}}^{\sigma} e^{-\left(\sigma^{\prime}-\mu_{G}\right)^{2} / 2 \delta_{G}^{2}} \mathrm{~d} \sigma^{\prime} \quad\left(\sigma>\sigma_{g r}\right)
\end{gathered}
$$

where $m, s_{0}=$ Weibull modulus and scale parameter of the Weibull tail, respectively, $\mu_{G}, \delta_{G}=$ mean and standard deviation of the Gaussian portion, $\sigma_{g r}=$ grafting stress at which the Weibull tail terminates, $P_{g r}=F_{f_{t}}\left(\sigma_{g r}\right)=$ grafting probability, and $r_{f}=$ re-scaling parameter. Among these six parameters, only four parameters are independent and the remaining two can be determined by the condition $F_{f_{t}}(\infty)=1$ and the continuity of the corresponding pdf at the grafting point, i.e. $\mathrm{d} F_{f_{t}}(\sigma) /\left.\mathrm{d} \sigma\right|_{\sigma_{g r}^{-}}=\mathrm{d} F_{f_{t}}(\sigma) /\left.\mathrm{d} \sigma\right|_{\sigma_{g r}^{+}}$. Previous studies $[5,4,21]$ demonstrated that the grafting probability is typically on the order of $10^{-4}-10^{-2}$.

On the continuum scale, the random strength field can be represented by a stationary process. The correlation function of the random strength field $f_{t}(x)$ is considered to follow an exponential form, i.e. $R_{f_{t}}(\Delta x)=\exp \left[-\left(\Delta x / l_{a}^{2}\right)\right]$, where $l_{a}=$ a length constant, and $\Delta x=\left|x_{2}-x_{1}\right|$. We choose $l_{a}=20 \mathrm{~nm}$, which is approximately on the order of the size of a single Si grain. The generation of the random strength field involves two steps: 1) generation of a standard Gaussian process $y(x)$ with a certain correlation function $\rho_{y}(\Delta x)$ and 2) transform the Gaussian variable to the target GaussWeibull variable, i.e. $f_{t}(x)=F_{f_{t}}^{-1}\left\{\Phi_{G}[y(x)]\right\}$. Note that the correlation function of the standard Gaussian process needs to be numerically determined to match the target correlation function of $f_{t}(x)$ at each value of $\Delta x$ through the following equation [16]:

$$
R_{f_{t}}(\Delta x)=\int_{-\infty}^{\infty} \int_{-\infty}^{\infty} F_{f_{t}}^{-1}\left[\Phi_{G}\left(y_{1}\right)\right] F_{f_{t}}^{-1}\left[\Phi_{G}\left(y_{2}\right)\right] \phi_{y_{1} y_{2}}\left[y_{1}, y_{2}, \rho_{y}(\Delta x)\right] \mathrm{d} y_{1} \mathrm{~d} y_{2}
$$

where $\phi_{y_{1} y_{2}}\left[y_{1}, y_{2}, \rho_{y}(\Delta x)\right]=$ joint pdf of standard Gaussian variables $y_{1}=y\left(x_{1}\right), y_{2}=y\left(x_{2}\right)$, i.e.

$$
\phi_{y_{1} y_{2}}\left[y_{1}, y_{2}, \rho_{y}(\Delta x)\right]=\frac{1}{2 \pi \sqrt{1-\rho_{y}^{2}(\Delta x)}} \exp \left\{-\frac{y_{1}^{2}+y_{2}^{2}-2 \rho_{y}(\Delta x) y_{1} y_{2}}{2\left[1-\rho_{y}^{2}(\Delta x)\right]}\right\}
$$


Fig. 4 shows the comparison between the correlation function of the original Gauss-Weibull random strength field and that of the transformed standard Gaussian field. It is seen that the correlation functions of these two fields are almost identical to each other. This indicates that the Weibull tail of the original strength distribution has a minimal effect on the autocorrelation feature of the transformed Gaussian variable.

By knowing the zero-mean processes $Z_{0}(x)$ and $f_{t 0}(x)$, we can obtain the process $\eta_{0}(x)$. Following Eqs. 6 to 13, we can use the statistical information of $\eta_{0}(x)$ to calculate the mean crossing rate $\mu_{\lambda}$ and therefore the probability distribution of the overall tensile strength of the specimen.

The aforementioned procedure is used to perform optimum fitting of the measured strength distributions of poly-Si tensile specimens with gauge lengths $L_{g}=20 \mu \mathrm{m}$ and $L_{g}=70 \mu \mathrm{m}$. Note that the uniaxial tensile specimens have two sidewalls (Fig. 1), which are statistically independent. Therefore, the strength distribution of the specimen can be calculated as

$$
\begin{aligned}
F\left(\sigma_{N}\right) & =1-\left\{F_{\eta_{0}}(\lambda) \exp \left[-\frac{\mu_{\lambda}}{F_{\eta_{0}}(\lambda)} L_{g}\right]\right\}^{2} \\
& =1-\left[F_{\eta_{0}}(\lambda)\right]^{2} \exp \left[-\frac{\mu_{\lambda} L}{F_{\eta_{0}}(\lambda)}\right]
\end{aligned}
$$

where the total sidewall length $L=2 L_{g}$. Fig. 5 shows that the present model matches the experimental results very well, and the corresponding fitted parameters for the strength cdf are $\mu_{G}=19 \mathrm{GPa}, \delta_{G}=$ $2.85 \mathrm{GPa}, P_{g r}=0.095, m=64, s_{0}=15.6 \mathrm{GPa}$, and $r_{f}=1.05$. It should be pointed out that the calibrated mean strength of silicon is similar to the theoretical strength of silicon predicted by ab initio simulations [10]. This indicates that the assumed length scales $\left(l_{x}\right.$ and $\left.l_{y}\right)$ used for stress averaging (Eqs. 1 and 2) and the autocorrelation length of the random strength field are on the right order of magnitude.

It is noted that the calibrated Weibull modulus $m$ is quite high. Recent studies $[4,21]$ showed that the strength cdf of brittle and quasibrittle materials can be derived from a statistical hierarchical model consisting bundles and chains, which predicts that the Weibull modulus increases from 2 at the nanoscale to some large number at the macroscale. So far it is unclear why the calculated Weibull modulus for Si materials at this small scale is so high. One possible reason is that there exists intrinsic randomness of microstructural geometries, which may raise the Weibull modulus. For example, Freudenthal [15] demonstrated that the Weibull modulus of material strength could be related to the probability distribution of the flaw size. One potential method for investigating this problem for Si materials is to employ an atomistic computational model to realistically simulate various nonlinear failure mechanisms with the information of the microstructural randomness of Si materials.

\section{Analysis of Failure Statistics of Flexural Specimens}

We now apply the model with the aforementioned calibrated parameters to predict the failure statistics of poly-Si MEMS beams under three-point bending. We consider geometrically similar beams of four different sizes, i.e. span-to-depth ratio $L / D=4$ and $L=10,20,40,80 \mu \mathrm{m}$. The beam attains its peak load once failure initiates from the bottom sidewall of the beam, which is governed by the same strength criterion (Eqs. 1 and 2). Here we denote the nominal strength of the beam as $\sigma_{m}=P_{\max } / b D$. With the stress gradient induced by the loading configuration, it is clear that the dimensionless stress field $Z(x)$ is non-stationary.

As mentioned in Section 3, in order to simplify the analysis, we first check whether the process $Z(x)$ can be approximated by a uniformly modulated process. For each beam size, we perform stochastic simulation of the elastic stress field, in which the random sidewall geometry is described by the aforementioned probability distributions of notch angle, notch depth, and notch spacing (Eqs. 24-26). After obtaining the random dimensionless stress field $Z(x)$, we compute its mean value $\mu_{z}(x)$ and extract the underlying zero-mean process $Z_{n}^{0}(x)$ based on Eq. 16. 
Note that here we consider a set of geometrically similar specimens and the specimen dimension is considerably larger than the characteristic size of the individual V-notches. Therefore, it is expected that we can use a single function $\mu_{z}(x / L)$ to describe the spatial variation of the mean value of $Z(x)$ for these specimens. Fig. 6a shows the simulated $\mu_{z}(x / L)$ for specimens of these four sizes, and it is seen that they are very close to each other. Likewise, it is found that the corresponding zero-mean process $Z_{n}^{0}(x)$ for these specimens could be described by a single stochastic process.

The stationarity of $Z_{n}^{0}(x)$ is first examined by calculating its covariance function along the specimen length. As a demonstration, Fig. 6b shows that the covariance function for the simulated $Z_{n}^{0}(x)$ for specimen of $L=10 \mu \mathrm{m}$. It is seen, for each gap distance $\Delta x$, the value of the covariance function fluctuates around a constant value regardless of coordinate $x$, in which the fluctuation is due to the finite-size sampling of the Monte Carlo simulations. Furthermore, Fig. 6c shows the ergodicity evaluation of $Z_{n}^{0}(x)$ by using Eq. 5. Figs. 6b) and c) indicate that $Z_{n}^{0}(x)$ can be considered as an ergodic stationary process.

To calculate the strength distributions of these geometrically similar beams, we average the mean value function $\mu_{z}(x / L)$ and obtain a single random process $Z_{n}^{0}(x)$ from the simulated random stress fields of specimens of all four sizes. By using Eq. 20, we obtain the strength distributions of these beams, as shown in Fig. 7. It is clearly seen that there exists a size effect on the strength cdf. It is interesting to note that all the simulated strength cdfs consist of a Weibull left tail (i.e. a powerlaw tail represented by dash lines in Fig. 7) with the same Weibull modulus. As the specimen size increases, the Weibull portion of the strength distribution extends, and eventually we would expect that the entire strength cdf would follow the Weibull distribution. Such size dependence of the strength distribution will be explained in detail in the next section.

So far there is a lack of experimental data on the strength distribution of poly-Si MEMS beams under three-point bending. In order to validate the present model for this loading case, we compare the strength distribution of specimen of $L=10 \mu \mathrm{m}$ predicted by the present model with that simulated by the Monte Carlo simulation, as shown in Fig. 8. It is seen that the present model agrees well with the Monte Carlo simulation results, which indicates that the present simplified analysis using the uniformly modulated process is sufficient for three-point bend MEMS specimens.

\section{Size Effect on Failure Probability}

Based on the foregoing analysis, it is evident that a salient feature of the present model is that it predicts a size effect on the failure probability of the MEMS specimen. This size effect is manifested not only in the statistical moments of the distribution function (e.g. mean and variance), but also in the functional form of the distribution function. In this section, we discuss the asymptotic behaviors of the strength distribution by using the aforementioned uniaxial tension specimen as an example.

Fig. 9 plots on the Weibull scale the calculated strength distribution of uniaxial tensile poly-Si specimens of different sidewall lengths $L$. It can be seen that, when $L$ is small, the far-left tail of the distribution function follows a Weibull cdf (equivalent to a power-law) and the majority part of the distribution deviates significantly from the Weibull cdf. For large $L$, the strength distribution approaches the two-parameter Weibull distribution, which is signified by a straight line on the Weibull plot. It is clear that, longer the specimen is, more potential failure locations the specimen would have. Therefore, it is expected that, as the specimen size becomes large, the strength distribution of the entire specimen is governed by the far-left tail of the failure statistics of each material point. This is the underlying idea of the classical theory of extreme value statistics, commonly referred to as the domain of attraction $[13,17,25]$. Consequently, to understand the aforementioned behaviors, it is essential to explore the tail behavior of the strength distribution.

We first study the asymptotic behavior of the cdf of $\eta_{0}(\lambda)$ for small stresses. Note that $F_{\eta_{0}}(\lambda)=$ $1-\operatorname{Pr}\left(\sigma_{N} Z \geq f_{t}\right)$. Instead of using the convolution (Eq. 8), it is easier to use the ratio of $f_{t}$ and $Z$ 
to compute this failure probability:

$$
\operatorname{Pr}\left(f_{t} / Z \leq \sigma_{N}\right)=\int_{0}^{\infty} F_{f_{t}}\left(\eta \sigma_{N}\right) f_{Z}(\eta) \mathrm{d} \eta
$$

Following Eq. 27a, the left-tail of $F_{f_{t}}(\sigma)$ is a power law. Since the random variable $Z$ is essentially bounded $\left(Z \in\left[1, z_{\max }\right]\right)$ (Fig. 3a), therefore for small values of $\sigma_{N}$ (i.e. $\sigma_{N}<x_{g r} / z_{\max }$ ), Eq. 32 can be written as

$$
F_{\eta_{0}}(\lambda)=1-\left(\frac{\sigma_{N}}{s_{1}}\right)^{m}
$$

where $s_{1}=s_{0}\left[\int_{0}^{\infty} \eta^{m} f_{z}(\eta) \mathrm{d} \eta\right]^{-1 / m}$.

Note that a small applied stress implies a high crossing barrier $\lambda$ and therefore a low crossing rate $\mu_{\lambda}$. Based on Eqs. 30 and 33, the strength distribution of the uniaxial tension specimen under small stresses can be rewritten as

$$
F\left(\sigma_{N}\right)=2\left(\frac{\sigma_{N}}{s_{1}}\right)^{m}+\frac{\delta_{\dot{\psi_{0}}}}{\sqrt{2 \pi}} \phi\left[\lambda_{G}\left(\sigma_{N}\right)\right] L \quad \text { as } \sigma_{N} \rightarrow 0
$$

As discussed in Section 3, the mean crossing rate $\mu_{\lambda}$ can be calculated through the transformation of $\eta_{0}$ to an equivalent standard Gaussian variable $\psi_{0}$ (Eq. 11), i.e.:

$$
\phi\left[\lambda_{G}\left(\sigma_{N}\right)\right]=\frac{1}{\sqrt{2 \pi}} \exp \left\{-\frac{1}{2}\left\{\Phi^{-1}\left[1-\left(\sigma_{N} / s_{0}\right)^{m}\right]\right\}^{2}\right\}
$$

The computation of $\delta_{\dot{\psi}_{0}}$ involves the evaluation of the power spectral density of the equivalent standard Gaussian process $\psi_{0}(x)$. It seems infeasible to obtain a closed form expression of $\delta_{\dot{\psi}_{0}}$ as a function of $\sigma_{N}$. In this study, we calculate the dependence of $\delta_{\dot{\psi}_{0}} \phi\left[\lambda_{G}\left(\sigma_{N}\right)\right]$ on $\sigma_{N}$, as shown in Fig. 10. Interestingly, it is seen that this dependence follows a power-law with an exponent $m$ for small $\sigma_{N}$, i.e. $\delta_{\dot{\psi}_{0}} \phi\left[\lambda_{G}\left(\sigma_{N}\right)\right] \propto \sigma_{N}^{m}$. With Eq. 34, we can conclude that

$$
F\left(\sigma_{N}\right) \propto \sigma_{N}^{m} \quad\left(\sigma_{N} \rightarrow 0\right)
$$

For large-size specimens, there are a vast number of potential failure locations, and the failure statistics of these locations is correlated. We can group these failure locations in such a way that the failure statistics of each group becomes statistically independent. Each group of these failure locations spans a length of $L_{0}$. Based on the foregoing analysis, it is clear that the strength distribution of each group would have a power-law tail, i.e. $P_{L_{0}}\left(\sigma_{N}\right)=\left(\sigma_{N} / s_{0 w}\right)^{m}$ as $\sigma_{N} \rightarrow 0\left(s_{0 w}=\right.$ constant $)$. At the large-size limit, the specimen still contains a large number of these groups, and according to the theory of extreme value statistics $[13,17]$, the strength distribution must follow the two-parameter Weibull distribution, i.e.:

$$
F\left(\sigma_{N}\right)=1-\exp \left[-\left(\sigma_{N} / s_{w}\right)^{m}\right]
$$

where $s_{w}=s_{w 0}\left(L_{0} / L\right)^{1 / m}=$ Weibull scaling parameter.

At the small-size limit (i.e. $L \rightarrow 0$ ), the strength distribution is primarily governed by the probability distribution of $\eta_{0}$ itself, i.e. $F\left(\sigma_{N}\right) \approx 1-F_{\eta_{0}}^{2}(\lambda)$. As indicated by Eq. 33, it has a power law tail and its extent is short. The bulk part of the strength distribution is difficult to determine. Fig. 11 plots $F\left(\sigma_{N}\right)$ for $L \rightarrow 0$ in a Gaussian distribution paper. It can be seen that the distribution reasonably follows a straight line for a probability larger than 0.2 . The transitional regime between the Weibull tail and the Gaussian part is very wide, and the functional form of the probability distribution in this regime is unclear. Nevertheless, this transition regime can be calculated accurately by the present model. For finite-size specimens, the lower part of the cdf has a Weibull cdf and the upper part deviates significantly from the Weibull distribution. As the specimen size increases, the Weibullian portion grows continuously and eventually occupies the entire distribution function, as indicated by Eq. 37. 
It should be emphasized that the aforementioned size dependence of strength cdf also applies to specimen of other geometries or under other loading configurations provided that the peak load is reached at the fracture initiation from the sidewalls. For example, the calculated size effect on the strength cdf of three-point bend specimens shown in Fig. 7 is in accordance with the foregoing analysis. It should be noted that the structural geometry and loading configurations would influence the stress field and therefore affect the behavior of the strength cdf for a given specimen. Consider two specimens of the same design but with different applied stress fields. We may set the strength distributions of these two specimens to be equal by changing the size of one of these specimens. Therefore, in the present model the effect of stress field on the strength distribution is equivalent to the size effect on the strength distribution. This is similar to the equivalence between specimen size and stress field in the context of the classical Weibull weakest-link model $[6,5,21]$.

\section{Size Effect on Mean Structural Strength}

The aforementioned size dependence of the failure statistics directly leads to a pronounced size effect on the mean structural strength. Similar to the foregoing analysis, we use the uniaxial tension specimen as an example to discuss the mean size effect behavior. The mean structural strength can be calculated as

$$
\bar{\sigma}_{m}=\int_{0}^{1} \sigma_{N} \mathrm{~d} F\left(\sigma_{N}\right)=\int_{0}^{\infty} 1-F\left(\sigma_{N}\right) \mathrm{d} \sigma_{N}
$$

We can determine the effect of the specimen size (or equivalently, the sidewall length) $L$ on the mean strength as shown in Fig. 12. Note that the small size limit $L \rightarrow 0$ in Fig. 12 is merely a mathematical abstraction since the smallest size of the specimen that is physically meaningful should be about the nominal width of the sidewall groove, which is approximately $50 \mathrm{~nm}$. Nevertheless, the plot of the entire mean size effect curve allows us to have a full understanding of the behavior of the present model.

It is interesting to note that the mean size effect curve approaches a horizontal small-size asymptote, i.e. $\bar{\sigma}_{m}=$ constant. Such an asymptote stems from the fact that the stationary stochastic process $\eta_{0}(x)$ has an intrinsic autocorrelation feature. Based on Eq. 6, the stochastic process $\eta_{0}(x)$ depends on $\sigma_{N}$, and therefore it is expected that, for each value of $\sigma_{N}$, there exists one autocorrelation length. The expectation of the autocorrelation length of $\eta_{0}(x)$ at the small-size limit can be calculated as

$$
L_{a}=\mathrm{E}\left[L_{\eta_{0}}\left(\sigma_{N}\right)\right]=\int_{0}^{\infty} L_{\eta_{0}}\left(\sigma_{N}\right) p\left(\sigma_{N}\right) \mathrm{d} \sigma_{N}
$$

where $L_{\eta_{0}}\left(\sigma_{N}\right)=$ autocorrelation length of $\eta_{0}(x)$ for a given value of $\sigma_{N}$, and $p\left(\sigma_{N}\right)$ is the pdf of $\sigma_{N}$ at the small size limit, i.e. $p\left(\sigma_{N}\right)=\mu_{z}^{-1} f_{\eta_{0}}\left(\mu_{f_{t}}-\sigma_{N} \mu_{z}\right)$.

It should be pointed out that the aforementioned definition of $L_{a}$ can also be extended to the case of three-point bending, where $\eta_{0}(x)$ should be replaced by $\eta_{n}(x)$ (Eq. 19). Since $\eta_{n}(x)$ is a nonstationary process, the autocorrelation length generally would vary along the beam length. In this case, we may define $L_{a}$ as the average of the spatially varying expectation of autocorrelation length, i.e.:

$$
L_{a}=\frac{1}{L} \int_{0}^{L} \int_{0}^{\infty} L_{\eta_{n}}\left(\sigma_{N}, x\right) p\left(\sigma_{N}\right) \mathrm{d} \sigma_{N} \mathrm{~d} x
$$

If the sidewall length of the specimen is smaller than a characteristic size $L_{p}\left(L_{p}=\gamma L_{a}, \gamma=\right.$ constant), the failure statistics of the each individual location of the sidewall starts to become statistically dependent. As a result, the mean structural strength would begin to approach a constant value, which is signified by a horizontal asymptote. It should be emphasized that $L_{p}$ represents a characteristic length scale of the present model, which governs the behavior of the failure statistics of the specimen. In this study, $L_{p}$ depends on several internal length scales, which include the autocorrelation length of the random field of the material strength and the autocorrelation length of the random stress field. 
The autocorrelation length of the random stress field is further related to the nonlocal averaging length scale $l_{x}$, which is a measure of the width of the FPZ and the probability distribution of the notch spacing. Therefore, the length scale $L_{p}$ of $\eta_{0}(x)$ contains the information of statistical length scale, geometrical length scale and fracture length scale (i.e. FPZ size). As will be shown in Section 8, the comparison between the present model and the conventional weakest-link model indicates that the length scale $L_{p}$ can approximately be set equal to the characteristic length $L_{0}$ introduced in Eq. 37 .

Furthermore, the present model also predicts the first order derivative of the mean size effect curve at the small-size limit:

$$
\bar{\sigma}_{m} \approx \int_{0}^{\infty} F_{\eta_{0}}^{2}\left(\sigma_{N}\right)\left[1-\frac{\mu_{\lambda}\left(\sigma_{N}\right)}{F_{\eta_{0}}\left(\sigma_{N}\right)} L\right] \mathrm{d} \sigma_{N}=\sigma_{0}-c_{1} L \quad(L \rightarrow 0)
$$

where $\sigma_{0}=\int_{0}^{\infty} F_{\eta_{0}}^{2}\left(\sigma_{N}\right) \mathrm{d} \sigma_{N}=$ strength at the small-size limit $(L \rightarrow 0)$ and $c_{1}=$ constant. It is interesting to note that this linear descent from the small-size strength limit is consistent with the small-size asymptotic behavior predicted by the cohesive crack model [3].

At the large-size limit, the strength distribution would necessarily approach the two-parameter Weibull distribution (Eq. 37). The corresponding mean strength can be calculated by using the well-known formula of the Weibullian mean, i.e.:

$$
\bar{\sigma}_{m}=s_{0 w} \Gamma\left(1+\frac{1}{m}\right)\left(\frac{L_{p}}{L}\right)^{1 / m}
$$

where $\Gamma(x)=$ Eulerian gamma function. Note that in Eq. $42 L_{0}$ is replaced by $L_{p}$. It is clear that this Weibull size effect would be reached as the strength distribution of the entire specimen is primarily governed by the power-law tail of specimen of size $L_{p}$. Consider that the power-law tail of specimen of length $L_{p}$ reaches a probability of $P_{t 0}$. In order to approach the Weibull asymptotic behavior, the strength distribution of the specimen need to follow the Weibull cdf up to some probability $P_{f w}$, and the corresponding specimen size should be at least on the order of

$$
L_{w}=-L_{p} \ln \left(1-P_{f w}\right) / P_{t 0}
$$

In the intermediate size range, there could exist another asymptote if $L_{p}$ and $L_{w}$ are far apart, which is usually the case. Such an asymptote is often termed as the intermediate asymptote [1]. The mean size effect could be described by a general power-law $\bar{\sigma}_{m} \propto L^{-1 / q}\left(L_{p} \ll L \ll L_{w}, q>0\right)$. This power-law exponent is governed by the statistical properties of the random process $\eta_{0}(x)$. For this regime of the mean size effect curve, the tail behavior of the random process $\eta_{0}(x)$ is unimportant.

To asymptotically match the aforementioned asymptotes, the following approximate equation can be used to describe the mean size effect for the entire size range:

$$
\bar{\sigma}_{m}=\sigma_{0}\left[\left(\frac{L_{p}}{L+L_{1}}\right)^{u}+\left(\frac{L_{2}}{L+L_{1}}\right)^{r / m}\right]^{1 / r}
$$

where $L_{p}, L_{1}, L_{2}, u, r=$ constants. Note that Eq. 44 consists of three asymptotes: 1) at the small size limit, $\bar{\sigma}_{m}=$ constant, 2$)$ in the intermediate size range, $\bar{\sigma}_{m}$ approaches another power law $\bar{\sigma}_{m} \propto$ $L^{-1 / q}(q=r / u)$, and 3$)$ at the large size limit, $\bar{\sigma}_{m}$ follows the classical Weibull size effect, i.e. $\bar{\sigma}_{m} \propto L^{-1 / m}$. It is clear that the characteristic length $L_{p}$ governs the transition from the horizontal small-size asymptote to the intermediate asymptote (see Fig. 12). Since the Weibull modulus is usually large, Eq. 44 becomes $\bar{\sigma}_{m}=\sigma_{0}\left(L_{w} / L\right)^{1 / m}$ as $L \rightarrow \infty$. Therefore, by matching the small and large size limits, we have

$$
\begin{aligned}
\left(L_{p} / L_{1}\right)^{u}+\left(L_{2} / L_{1}\right)^{r / m} & =1 \\
s_{0 w} \Gamma\left(1+\frac{1}{m}\right) L_{p}^{1 / m} & =\sigma_{0} L_{2}^{1 / m}
\end{aligned}
$$


Eq. 46 shows that $L_{2}$ is directly related to the length scale $L_{p}$. Furthermore, we note that the length constant $L_{2}$ can be directly related to the aforementioned length scale $L_{w}$, at which the Weibull asymptote is approached. In the context of the mean size effect curve, we may define $L_{w}$ as the specimen size at which the intermediate asymptote and the large-size asymptote intersects (Fig. 12). Therefore, we have $L_{2}=\left(L_{p} / L_{w}\right)^{m / q} L_{w}$. Substitution of this relationship into Eq. 45 yields $\left(L_{p} / L_{1}\right)^{u}+\left(L_{p} / L_{w}\right)^{r / q}\left(L_{w} / L_{1}\right)^{r / m}=1$. Since $L_{p} \ll L_{w}$ and $r / m \ll 1$, we may conclude that $\left(L_{p} / L_{w}\right)^{r / q}\left(L_{w} / L_{1}\right)^{r / m} \ll\left(L_{p} / L_{1}\right)^{u}$, which implies that $L_{p} \approx L_{1}$. Therefore, it is clear that all the length constants introduced in this approximate size effect equation are related to the characteristic length scale $L_{p}$.

Fig. 12 shows that Eq. 44 agrees well with the simulated mean size effect curve of the MEMS specimens under uniaxial tension. Based on the fitting, we obtain $L_{p} \approx 70 \mathrm{~nm}$. As discussed earlier in Section 4, the autocorrelation lengths of the dimensionless stress field $Z(x)$ and the material strength field $f_{t}(x)$ are $100 \mathrm{~nm}$ and $50 \mathrm{~nm}$, respectively, and the characteristic autocorrelation length $L_{a}$ of the process $\eta_{0}(x)$ should lie between these two values. Therefore, for the uniaxial tensile specimen studied here, we expect that the proportionality constant $\gamma$ that relates $L_{p}$ and $L_{a}$ would be on the order of 1.

It should be pointed out that, in the existing literature of quasibrittle fracture, the size effect of this kind was usually regarded as energetic-statistical in nature $[2,3,5]$. The conventional weakest-link model is unable to yield a horizontal small-size asymptote since the specimen size cannot be smaller than the size of the material element that is represented by each link. As a result, the horizontal small-size asymptote needs to be derived separately from the cohesive crack model, which yields a plastic behavior at the small-size limit and therefore a vanishing size effect. This asymptotic behavior is then superimposed onto the statistical size effect predicted by the weakest-link model, which leads to an energetic-statistical scaling law $[2,3]$.

By contrast, in this study we show that the entire mean size effect curve can be described by a pure probabilistic model. In other words, this type of the size effect can be obtained without relying on any nonlinear material constitutive models. The essence is to formulate a model that contains the spatial autocorrelation feature of the failure statistics, which in principle could deal with specimens of any size.

\section{Relation with Finite Weakest-Link Model of Structural Strength}

It is worthwhile to compare the present model with the conventional weakest-link model in the context of this study. The weakest-link model is the most widely used statistical model for the strength of brittle and quasibrittle structures. In the weakest-link model, the structure is considered to be represented by a chain of statistically independent elements, in which the failure of one single element would trigger the failure of the entire structure (under a controlled load). Therefore, it is clear that the weakest-link model is essentially a discrete model, in which the size of each material element represents an essential length scale for the discretization. By contrast, the present model is anchored by a continuous description of the failure statistics, which contains a length scale $L_{p}$ that represents the statistical autocorrelation feature of the failure of material points.

First consider the case of uniaxial tension specimen in which we adopt the concept of stationary process. In the spirit of the finite weakest-link model, the overall strength distribution of the specimen (Eq. 7) can be rewritten as

$$
\begin{aligned}
F\left(\sigma_{N}\right) & =1-F_{\eta_{0}}^{2}\left[\lambda\left(\sigma_{N}\right)\right]\left\{\exp \left(-\frac{\mu_{\lambda}\left(\sigma_{N}\right) L_{0}}{F_{\eta_{0}}\left[\lambda\left(\sigma_{N}\right)\right]}\right)\right\}^{\frac{L}{L_{0}}} \\
& =1-\left[1-F_{1}\left(\sigma_{N}\right)\right]^{n}
\end{aligned}
$$


where $n=L / L_{0}$, and

$$
F_{1}\left(\sigma_{N}\right)=1-\left\{F_{\eta_{0}}\left[\lambda\left(\sigma_{N}\right)\right]\right\}^{2 / n} \exp \left(-\frac{\mu_{\lambda}\left(\sigma_{N}\right) L_{0}}{F_{\eta_{0}}\left[\lambda\left(\sigma_{N}\right)\right]}\right)
$$

Based on Eqs. 47 and 48, it is clear that the present model can be translated to a finite weakest-link model. Though Eqs. 47 does not pose any restrictions on length $L_{0}$, from a physical viewpoint $L_{0}$ should be larger than the length scale $L_{p}$ extracted from the characteristic autocorrelation length of the random process $\eta_{0}(x)$. If we further define $L_{0}$ as the minimum size of the statistically independent element, whose failure triggers the failure of the structure, then $L_{0}$ should be set equal to $L_{p}$.

Note that Eq. 49 indicates that $F_{1}\left(\sigma_{N}\right)$ is not exactly equal to the failure probability of a sidewall of length $L_{p}$, which can be written as

$$
F_{L_{p}}\left(\sigma_{N}\right)=1-F_{\eta_{0}}\left[\lambda\left(\sigma_{N}\right)\right] \exp \left(-\frac{\mu_{\lambda}\left(\sigma_{N}\right) L_{p}}{F_{\eta_{0}}\left[\lambda\left(\sigma_{N}\right)\right]}\right)
$$

The difference between Eq. 49 and 50 lies in the prefactor of the exponential term. As pointed out in $[24,25]$, this prefactor represents the non-crossing probability of the starting point of the process. In the present model, we have only two starting points for the two statistically independent sidewalls. In fact, Eq. 47 can be considered as a weakest-link model of $n+1$ elements, in which the first element represents the two starting points and rest of $n$ elements represent the material elements of length $L_{p}$ (Fig. 13). To make the model match the conventional weakest-link model, we can distribute the survival probability of the starting points evenly to $n$ elements, which leads to Eq. 49. It should be pointed out that one should not directly incorporate Eq. 50 into the weakest-link model (i.e. $\left.F_{L_{p}}\left(\sigma_{N}\right) \neq F_{1}\left(\sigma_{N}\right)\right)$ because for each sidewall, except for the first element, the starting point of each subsequent element is fully correlated to the end point of the previous element. Therefore, one cannot treat the failure statistics of every starting point as a statistically independent quantity.

The foregoing analysis can easily be extended to MEMS specimens of other geometries or under other loading configurations. Eq. 20 can be rewritten in the spirit of the weakest-link model, i.e.:

$$
\begin{aligned}
F\left(\sigma_{N}\right) & =1-F_{\eta_{0}}\left[\lambda_{0}\left(\sigma_{N}\right)\right] \prod_{i=1}^{n} \exp \left\{-\int_{x_{i}-L_{p} / 2}^{x_{i}+L_{p} / 2} \frac{\mu_{\lambda}(x)}{F_{\eta_{0 \mid x}}[\lambda(x)]} \mathrm{d} x\right\} \\
& =1-\prod_{i=1}^{n}\left[1-F_{1}\left(\sigma_{N}, x_{i}\right)\right]
\end{aligned}
$$

where

$$
F_{1}\left(\sigma_{N}, x_{i}\right)=1-\left\{F_{\eta_{0}}\left[\lambda_{0}\left(\sigma_{N}\right)\right]\right\}^{1 / n} \exp \left\{-\int_{x_{i}-L_{p} / 2}^{x_{i}+L_{p} / 2} \frac{\mu_{\lambda}(x)}{F_{\eta_{0 \mid x}}[\lambda(x)]} \mathrm{d} x\right\}
$$

Similar to the previous case, $F_{1}\left(\sigma_{N}, x_{i}\right)$ represents the failure probability of a material element centered at coordinate $x_{i}$ with a prefactor, which accounts for the survival probability at the maximum stress point distributed over all $n$ material elements.

Based on the aforementioned discussion, it has transpired that the present model is a generalization of the finite weakest-link model by incorporating the detailed statistical information of the failure distribution of each element. This information naturally contains the underlying autocorrelation features of the various relevant random fields. By contrast, the conventional weakest-link model assigns a single probability distribution function to each element without considering the detailed stochastic processes that govern the failure of the element. Furthermore, the present analysis also indicates that the material element size of the finite weakest-link model is related to the characteristic autocorrelation length of the random field that governs the material failure. This offers us a new insight into how to determine the material length scale of the finite weakest-link model. 
It should be emphasized that the present model is anchored by modeling the failure of material points as a 1-D stochastic process, which is consistent with the failure mechanism of MEMS specimens considered in this study. To apply the model to other quasibrittle structures, such as concrete, ceramics, rock, composites, etc., the present model needs to be extended to a higher dimension with a multi-dimensional first passage analysis. Similar to the present model, such a first passage model is expected to appear as a generalization of the weakest-link model for $2 \mathrm{D}$ or $3 \mathrm{D}$ problems, which will elucidate the physical meaning of the intrinsic length scale of the weakest-link model.

\section{Conclusions}

In this study, a first passage based probabilistic model is developed for the failure statistics of poly-Si MEMS structures. The model is able to realistically incorporate the detailed statistical information of random fields of both material strength and applied stress with nonlocal averaging. Depending on the specimen geometry and loading configuration, the failure statistics of each potential failure location can be modeled by either a stationary stochastic process or a uniformly modulated stochastic process. The overall failure risk of the structure can be evaluated through the first passage analysis. The model is validated by the optimum fitting of the measured strength distributions of uniaxial tensile poly-Si MEMS specimens of two gauge lengths.

It is shown that the present model naturally involves an intrinsic length scale, which is related to the characteristic autocorrelation length of the underlying random field governing the material failure. This length scale is determined by the autocorrelation length of the strength field, the geometrical randomness of the sidewall grooves, and the length scale for nonlocal stress averaging . The resulting strength distribution is strongly dependent on the specimen size relative to this length scale. For finite-size specimens, the strength distribution is highly non-Weibullian except for its far left tail. For large-size specimens, the strength distribution follows the two-parameter Weibull distribution, which is consistent with the theory of extreme value statistics. Such size dependence of the strength cdf further leads to a strong size effect on the mean strength. Due to the consideration of the autocorrelation features of the random stress and strength fields, the model is able to yield a horizontal small-size asymptote of the mean size effect curve. At the large-size limit, the model predicts the classical Weibull size effect. This result indicates that the entire mean size effect of this kind can be explained from a pure statistical viewpoint.

It is demonstrated that the present model can be translated to the conventional weakest-link model. Compared to the weakest-link model, the present model contains much richer information of the statistics of different parameters that governs the material failure. This information allows a better determination of the failure probability of each material element for the weakest-link model. Meanwhile, it is shown that the size of the material element required for the weakest-link model is directly related to the characteristic autocorrelation length of the present model.

Acknowledgment: The authors gratefully acknowledge the financial support under Grant NSF/CMMI1361868 to the University of Minnesota from the U.S. National Science Foundation.

\section{References}

[1] G. I. Barenblatt. Scaling. Cambridge University Press, Cambridge, 2003.

[2] Z. P. Bažant. Scaling theory of quaisbrittle structural failure. Proc. Nat'l. Acad. Sci., USA, 101(37):13400-13407, 2004.

[3] Z. P. Bažant. Scaling of Structural Strength. Elsevier, London, 2005. 
[4] Z. P. Bažant, J.-L. Le, and M. Z. Bazant. Scaling of strength and lifetime distributions of quasibrittle structures based on atomistic fracture mechanics. Proc. Nat'l. Acad. Sci., USA, 106:11484-11489, 2009.

[5] Z. P. Bažant and S. D. Pang. Activation energy based extreme value statistics and size effect in brittle and quasibrittle fracture. J. Mech. Phys. Solids., 55:91-134, 2007.

[6] Z. P. Bažant and J. Planas. Fracture and Size Effect in Concrete and Other Quasibrittle Materials. CRC Press, Boca Raton, 1998.

[7] B. L. Boyce, R. Ballarini, and I. Chasiotis. An argument for proof testing brittle microsystems in high-reliability applications. J. Micromech. Microeng., 18:117001, 2008.

[8] B. L. Boyce, J. M. Grazier, T. E. Buchheit, and M. J. Shaw. Strength distributions in polycrystalline silicon MEMS. J. Microelectromech. Syst., 16(2):179-190, 2007.

[9] G. Deodatis. Non-stationary stochastic vector processes: seismic ground motion applications. Prob. Engrg. Mech., 11:149-168, 1996.

[10] S. M.-M. Dubois, G.-M. Rignanese, T. Pardoen, and J.-C. Charlier. Ideal strength of silicon: An ab initio study. Phys. Rev. B, 74:235203, 2006.

[11] H. D. Espinosa, B. Peng, N. Moldovan, T. A. Friedmann, X. Xiao, D. C. Mancini, O. Auciello, J. Carlisle, and C. A. Zorman. A comparison of mechanical properties of three MEMS materialssilicon carbide, ultrananocrystalline diamond, and hydrogen-free tetrahedral amorphous carbon (Ta-C). In A. Carpinteri, editor, 11th International Conference on Fracture, volume 5, pages 3806-3811, 2005.

[12] F. J. Ferrante, S. R. Arwade, and L. L. Graham-Brady. A translational model for non-stationary, non-Gaussian random processes. Prob. Engrg. Mech., 20:215-228, 2005.

[13] R. A. Fisher and L. H. C. Tippett. Limiting form of the frequency distribution the largest and smallest number of a sample. Proc. Cambridge Philos. Soc., 24:180-190, 1928.

[14] A. M. Fitzgerald, D. M. Pierce, B. M. Huigens, and C. D. White. A general methodology to predict the reliability of single-crystal silicon MEMS devices. J. Microelectromech. Syst., 18(4):962-970, 2009 .

[15] A. M. Freudenthal. Statistical approach to brittle fracture. In Fracture: An Advanced Treatise, vol. 2, pages 591-619, New York, 1968.

[16] M. Grigoriu. Crossings of non-Gaussian translation processes. J. Engrg. Mech. ASCE, 110(4):610620, 1984.

[17] E. J. Gumbel. Statistics of Extremes. Columbia University Press, New York, 1958.

[18] S. S. Hazra, M. S. Baker, J. L. Beuth, and M. P. de Boer. Demonstration of an in-situ on-chip tester. J. Micromech. Microeng., 19:082001 (5 pp.), 2009.

[19] H. Kim and M.D. Shields. Modeling strongly non-gaussian non-stationary stochastic processes using the Iterative Translation Approximation method and Karhunen-Loeve expansion. Comp. Struct., 161:31-42, 2015.

[20] J.-L. Le, R. Ballarini, and Z. Zhu. Modeling of probabilistic failure of polycrystalline silicon MEMS structures. J. Amer. Cer. Soc., 98(6):1685-1697, 2015. 
[21] J.-L. Le, Z. P. Bažant, and M. Z. Bazant. Unified nano-mechanics based probabilistic theory of quasibrittle and brittle structures: I. Strength, crack growth, lifetime and scaling. J. Mech. Phys. Solids., 59:1291-1321, 2011.

[22] E. D. Reedy Jr. Singular stress fields at the intersection of a grain boundary and a stress-free edge in a columnar polycrystal. J. Appl. Mech. ASME, 78:014502, 2011.

[23] E. D. Reedy, Jr., B. L. Boyce, J. W. Foulk, III, R. V. Field Jr., M. P. de Boer, and S. S. Hazra. Predicting fracture in micrometer-scale polycrystalline silicon MEMS structures. J. Microelectromech. Syst., 20(4):922-932, 2011.

[24] E. Vanmarcke. On the distribution of the first-passage time for normal stationary random processes. J. Appl. Mech. ASME, 42:215-220, 1975.

[25] E. Vanmarcke. Random Fields Analysis and Synthesis. World Scientific Publishers, Singapore, 2010 .

[26] M. Vořechovský. Incorporation of statistical length scale into weibull strength theory for composites. Comp. Struct., 92(9):2027-2034, 2010.

[27] W. Weibull. The phenomenon of rupture in solids. Proc. Royal Sweden Inst. Engrg. Res., 153:1-55, 1939.

[28] W. Weibull. A statistical distribution function of wide applicability. J. Appl. Mech. ASME, 153(18):293-297, 1951.

[29] K. Yasutake, M. Iwata, K. Yoshii, M. Umeno, and H. Kawabe. Crack healing and fracture strength of silicon crystals. J. Mater. Sci., 21(6):2185-2192, 1986. 


\section{List of Figures}

1 Schematic of a uniaxial tensile MEMS specimen with sidewall surface grooves idealized by V-notches. . . . . . . . . . . . . . . . . . . . . . 20

2 Illustration of first passage analysis of failure of a uniaxial tension specimen. . . . . . . . 20

3 a) Underlying probability distribution of $Z(x)$, b) ergodicity check of the zero-mean process $Z_{0}(x)\left(\delta_{Z_{0}}=\right.$ standard deviation of $\left.Z_{0}\right)$, and c) correlation function of $Z_{0}(x) \ldots \ldots$

4 Comparison of the correlation functions of the stochastic process $f_{t}(x)$ and its equivalent standard Gaussian process. . . . . . . . . . . . . . . . . . . . . .

5 Optimum fits of the measured strength histograms of poly-Si tensile specimens by the present model. . . . . . . . . . . . . . . . . . . . . .

6 Properties of the uniformly modulated stochastic process $Z(x)$ for random stress field of the three-point bend beams: a) spatial variation of mean value of $Z(x)$ for different specimen sizes, b) covariance of the zero-mean stationary process $Z_{n}^{0}(x)$ for specimen size $L=10 \mu \mathrm{m}$, and c) ergodicity check of $Z_{n}^{0}(x)$ for specimen size $L=10 \mu \mathrm{m}\left(\delta_{Z_{n}^{0}}=\right.$ standard deviation



7 Predicted strength distributions of geometrically similar poly-Si beams of four different sizes. 21

8 Comparison of the strength distributions of beam of $L=10 \mu \mathrm{m}$ predicted by the present model and the Monte Carlo simulation. . . . . . . . . . . . . . . . . . . . 21

9 Size effect on the strength distribution of poly-Si MEMS specimens under uniaxial tension. 21

10 Numerically simulated relation between $\delta_{\dot{\psi}_{0}} \phi\left[\lambda_{G}\left(\sigma_{N}\right)\right]$ and $\sigma_{N} \ldots \ldots \ldots \ldots 21$

11 Probability distribution of $F\left(\sigma_{N}\right)(L \rightarrow 0)$ plotted in the Gaussian distribution paper. . . . 21

12 Size effect on the mean strength of poly-Si MEMS structures under uniaxial tension. . . . 21

13 Relation between the present model and the conventional weakest-link statistical model of structural failure. . . . . . . . . . . . . . . . . . . . . . . . . 21 

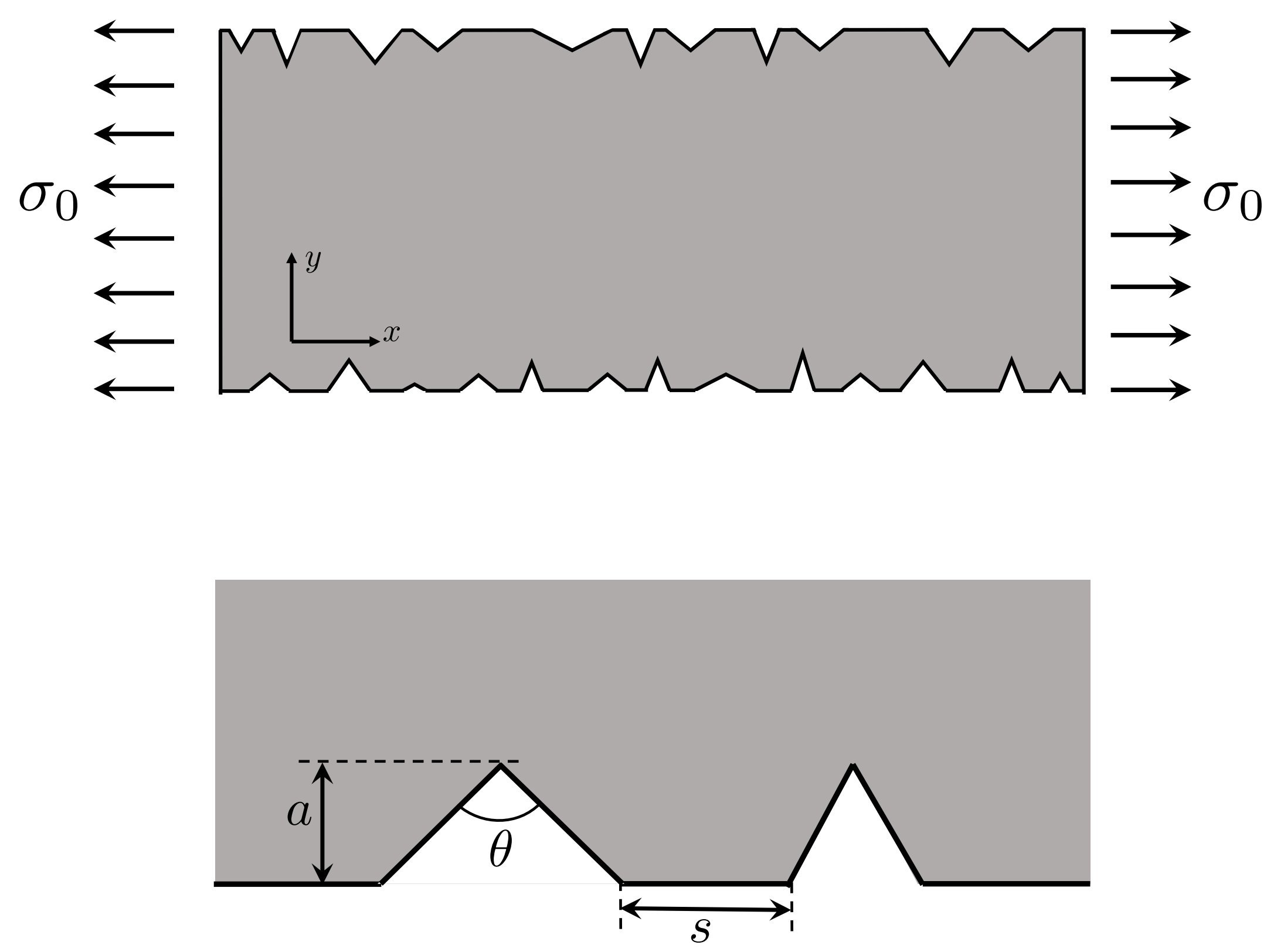

Fig. 1 


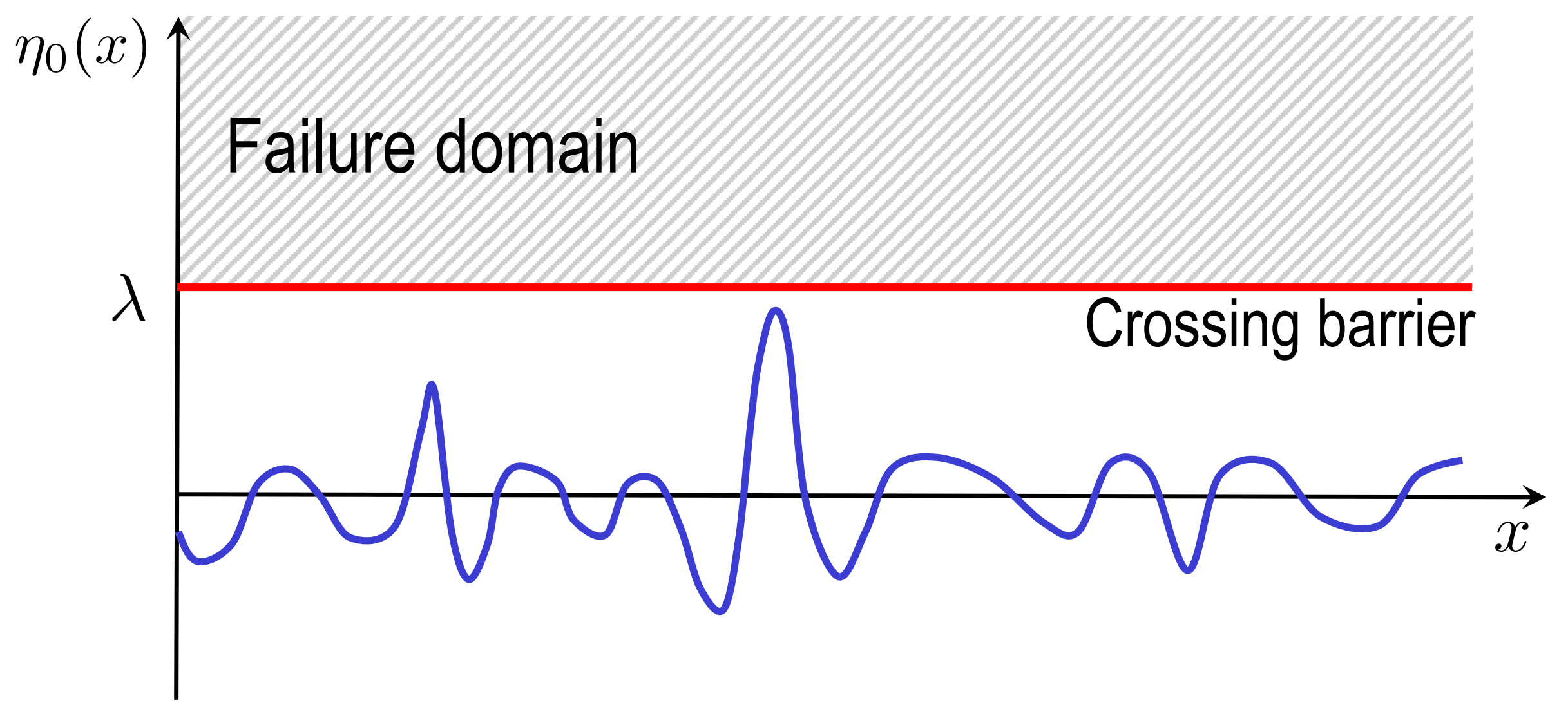

Fig. 2 

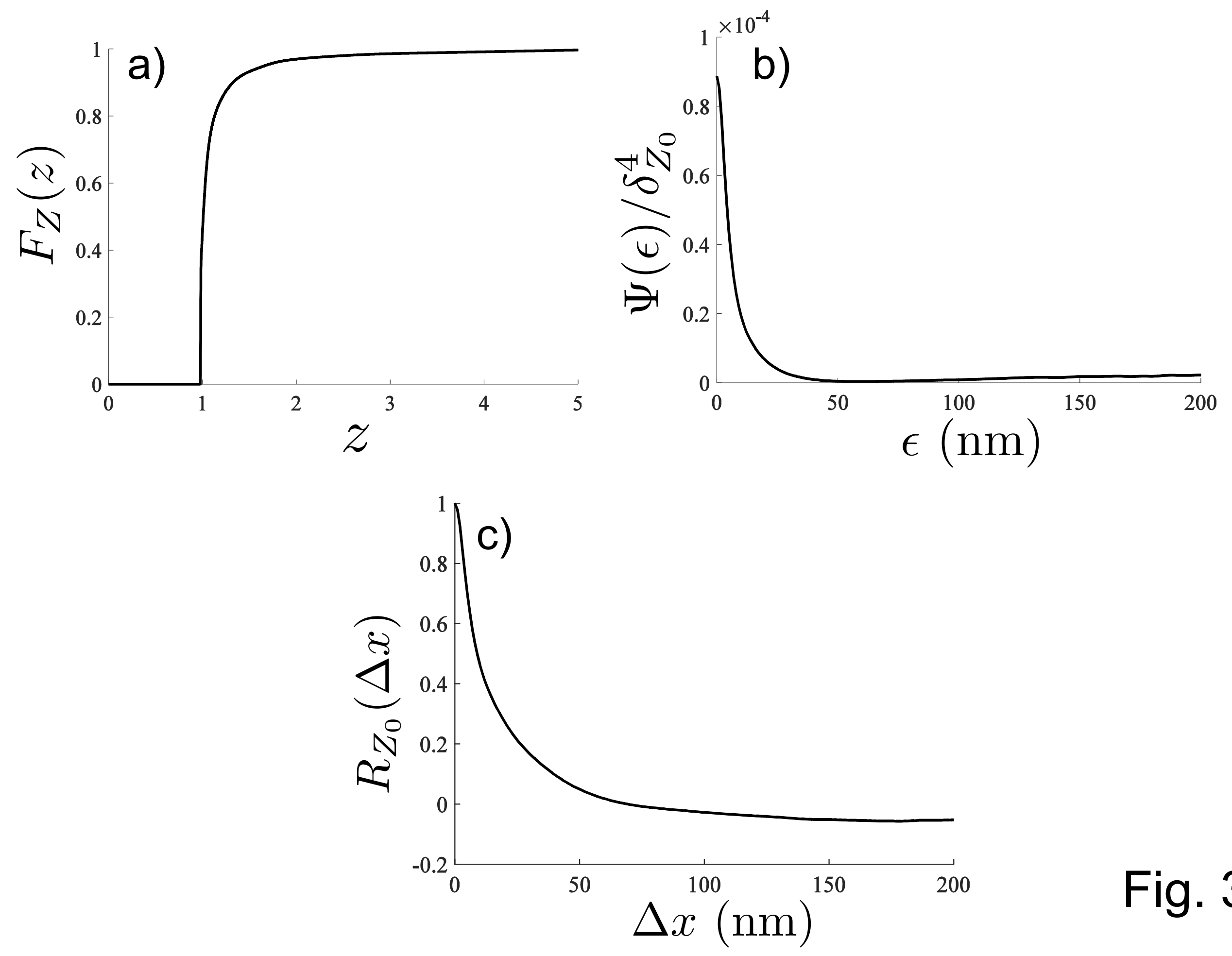

Fig. 3 




Fig. 4 

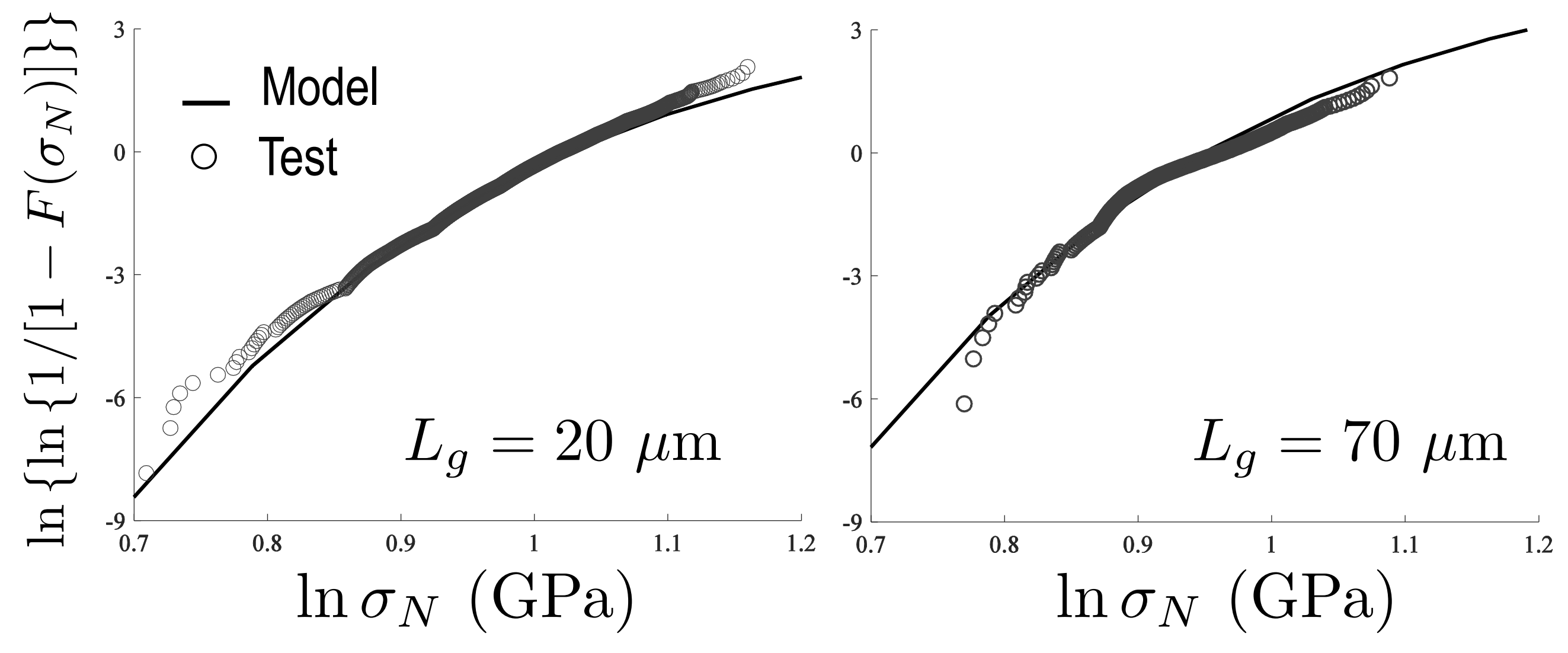

Fig. 5 

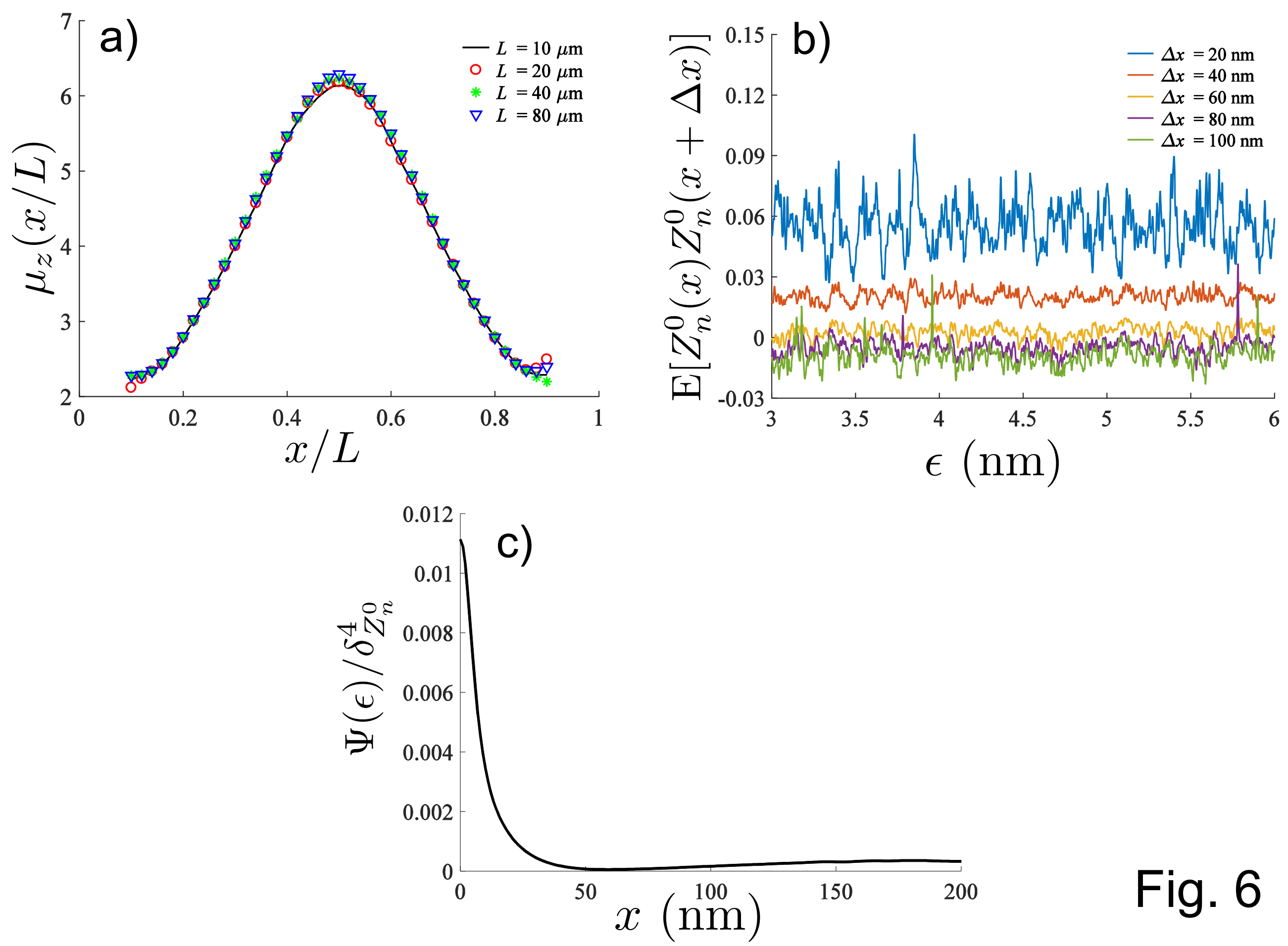

Fig. 6 


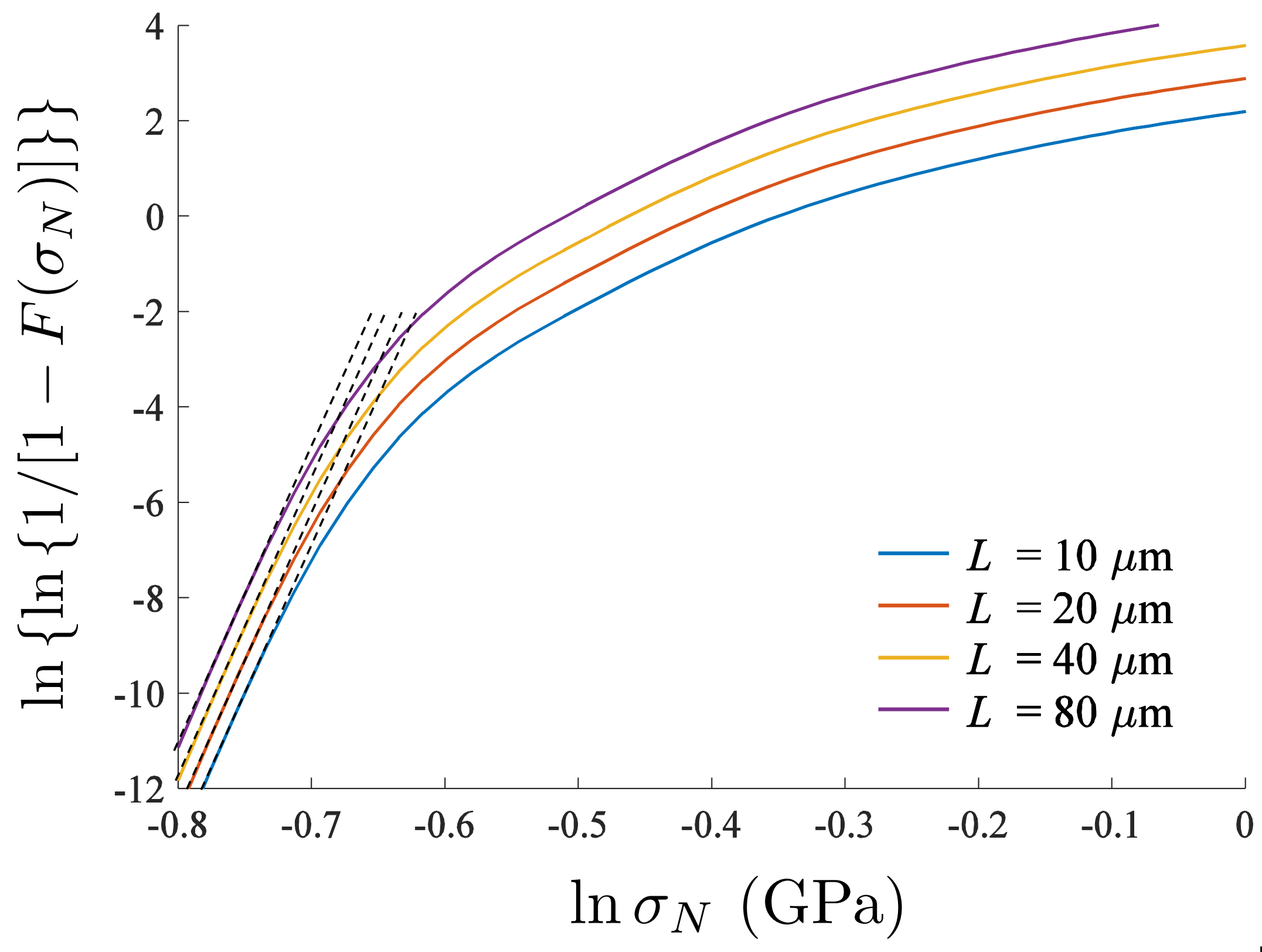

Fig. 7 


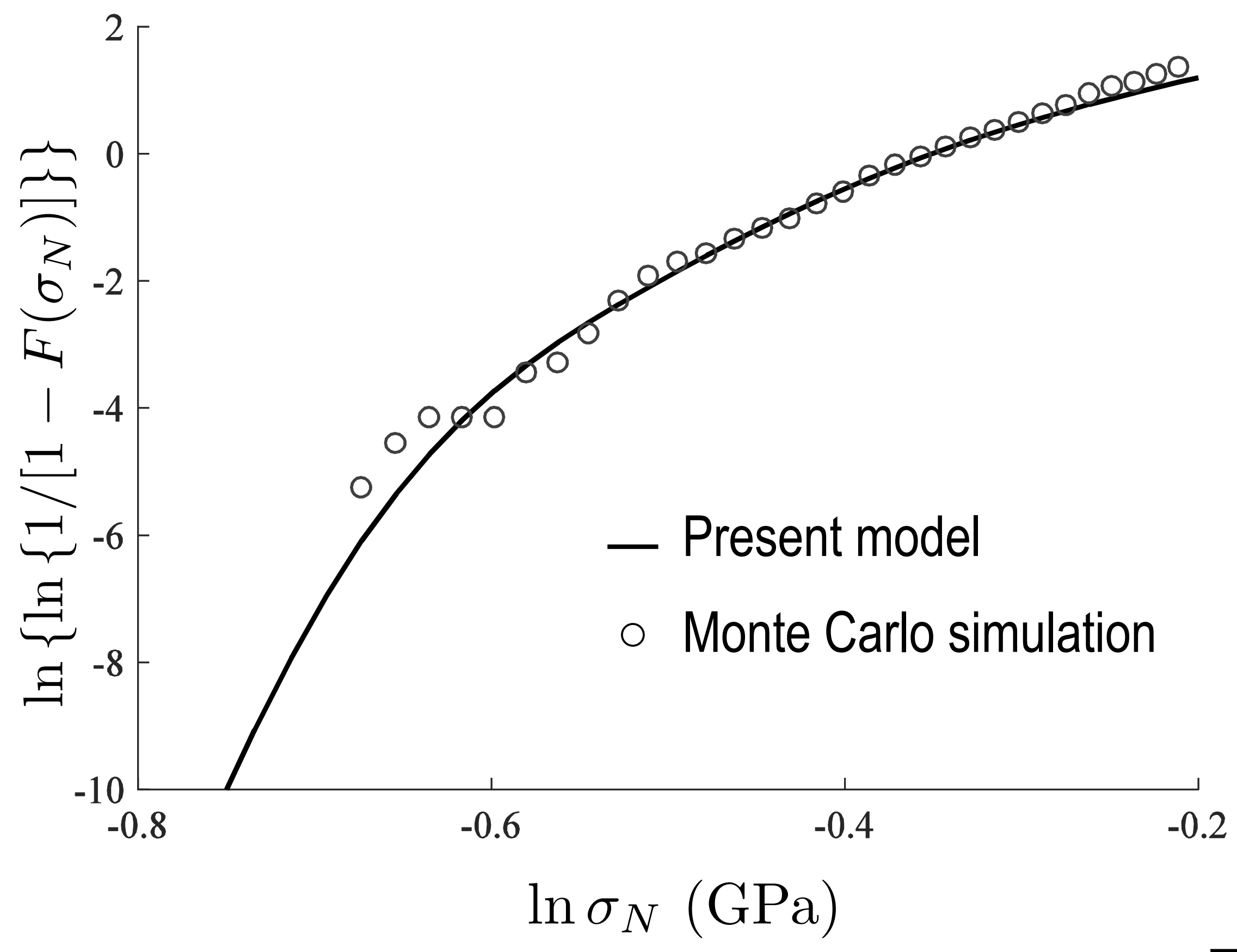

Fig. 8 


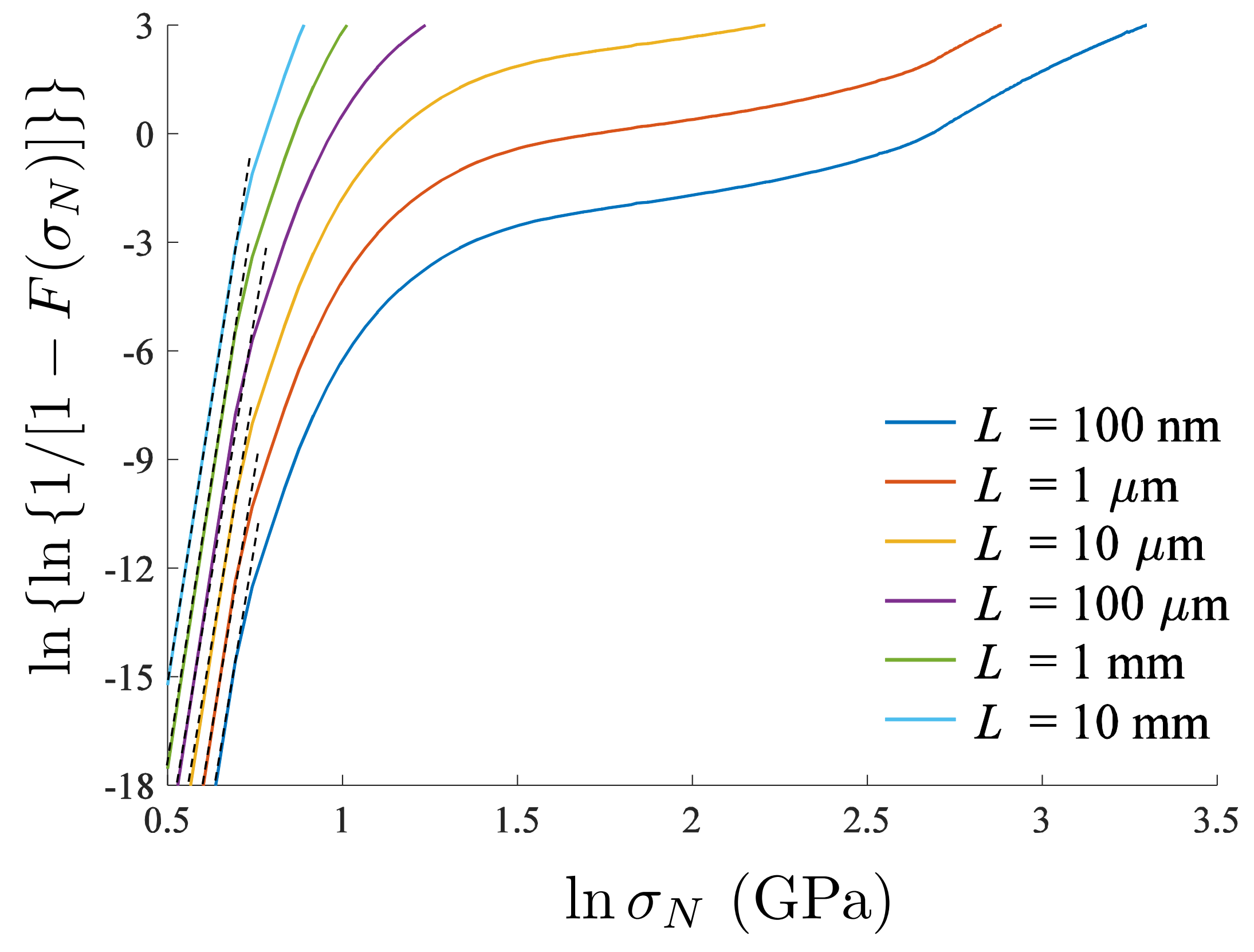

Fig. 9 


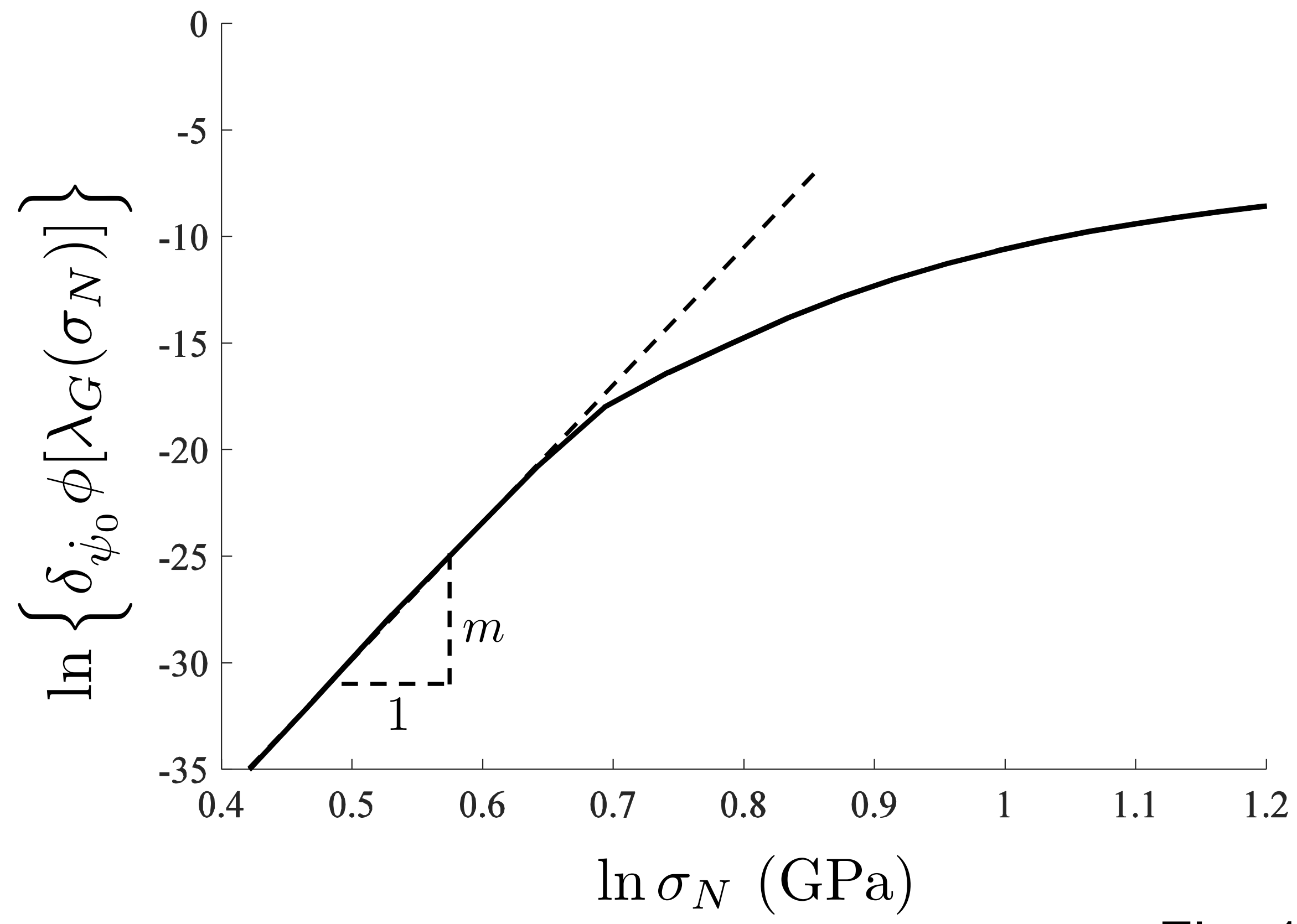

Fig. 10 


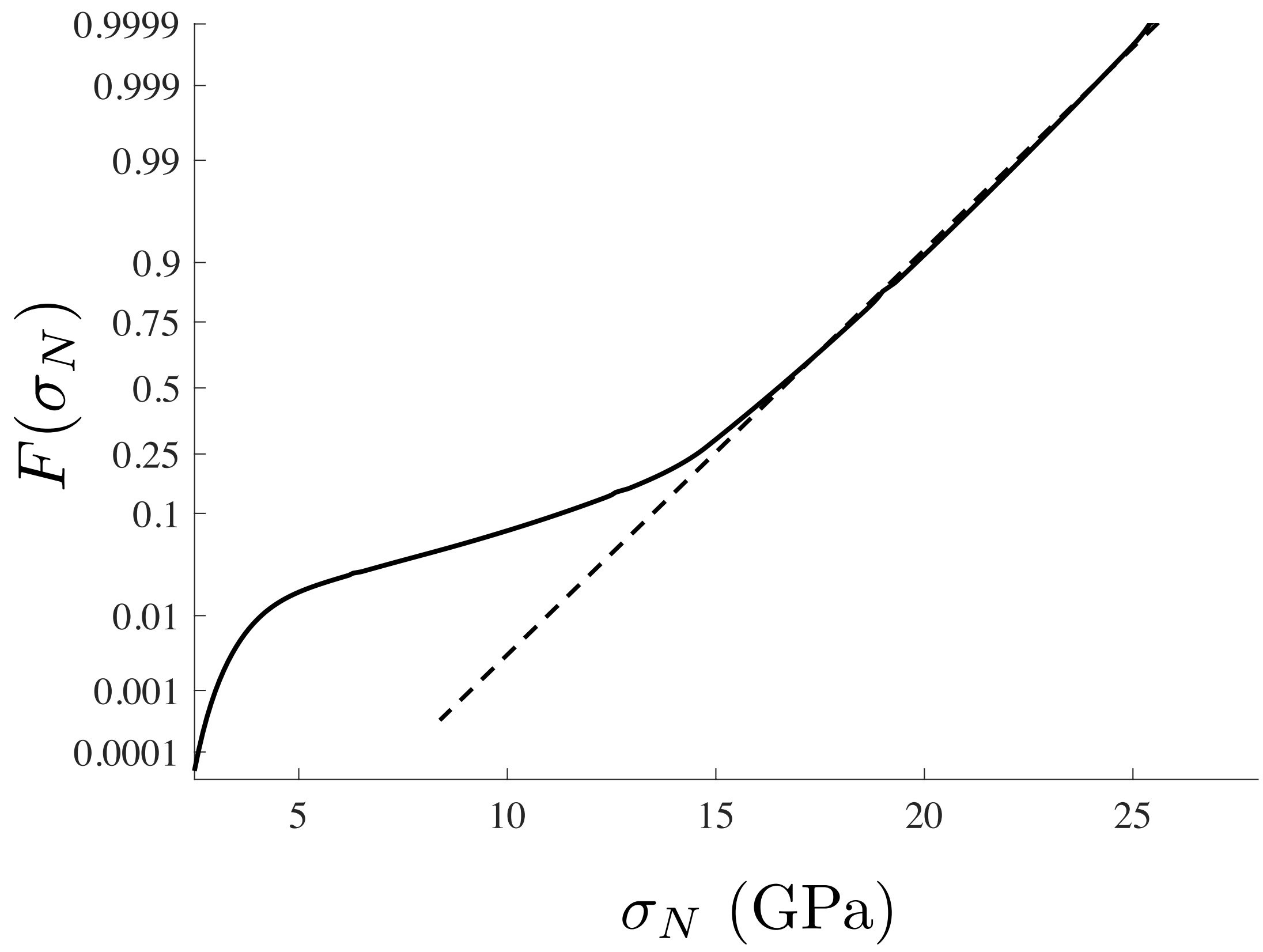

Fig. 11 


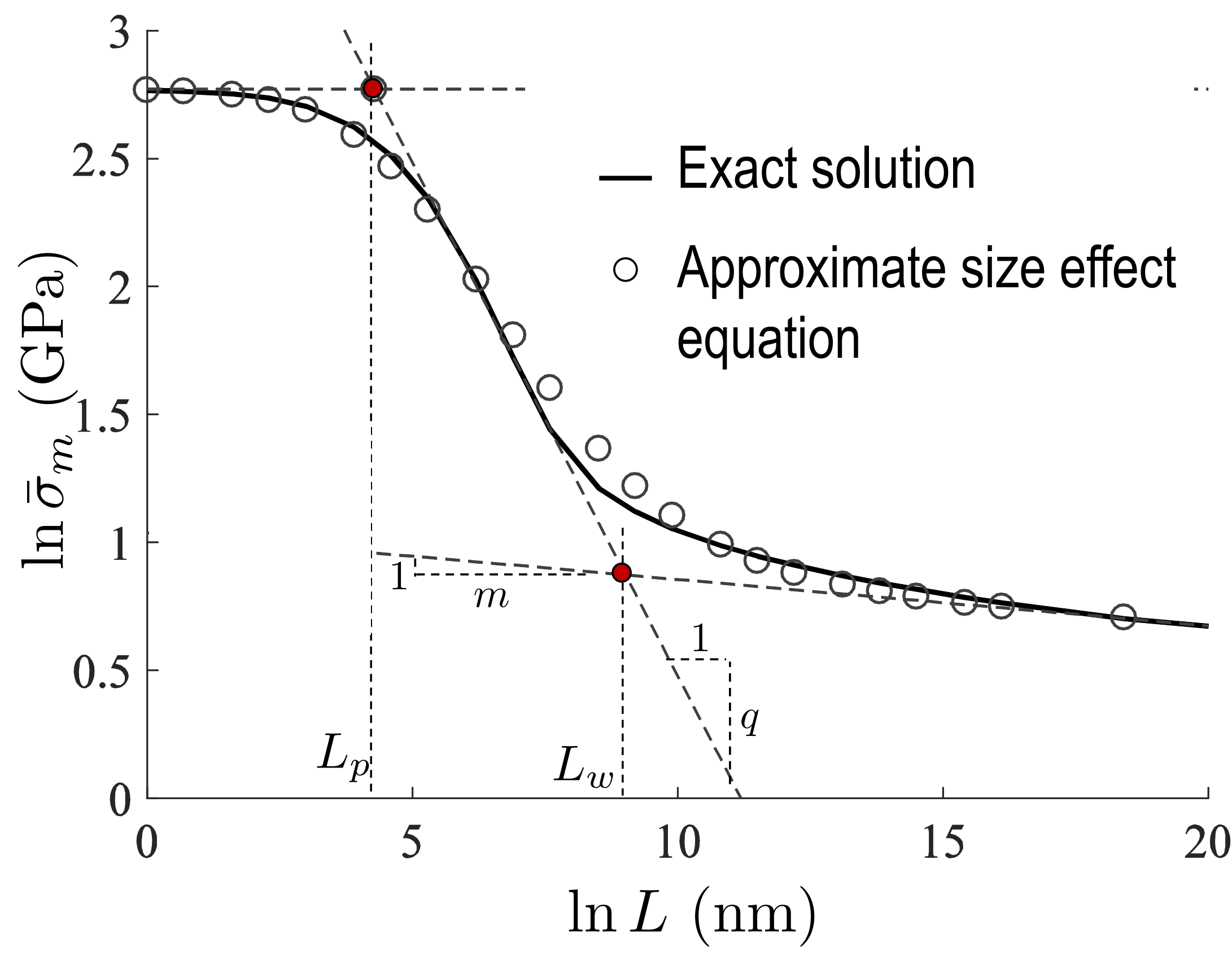

Fig. 12 

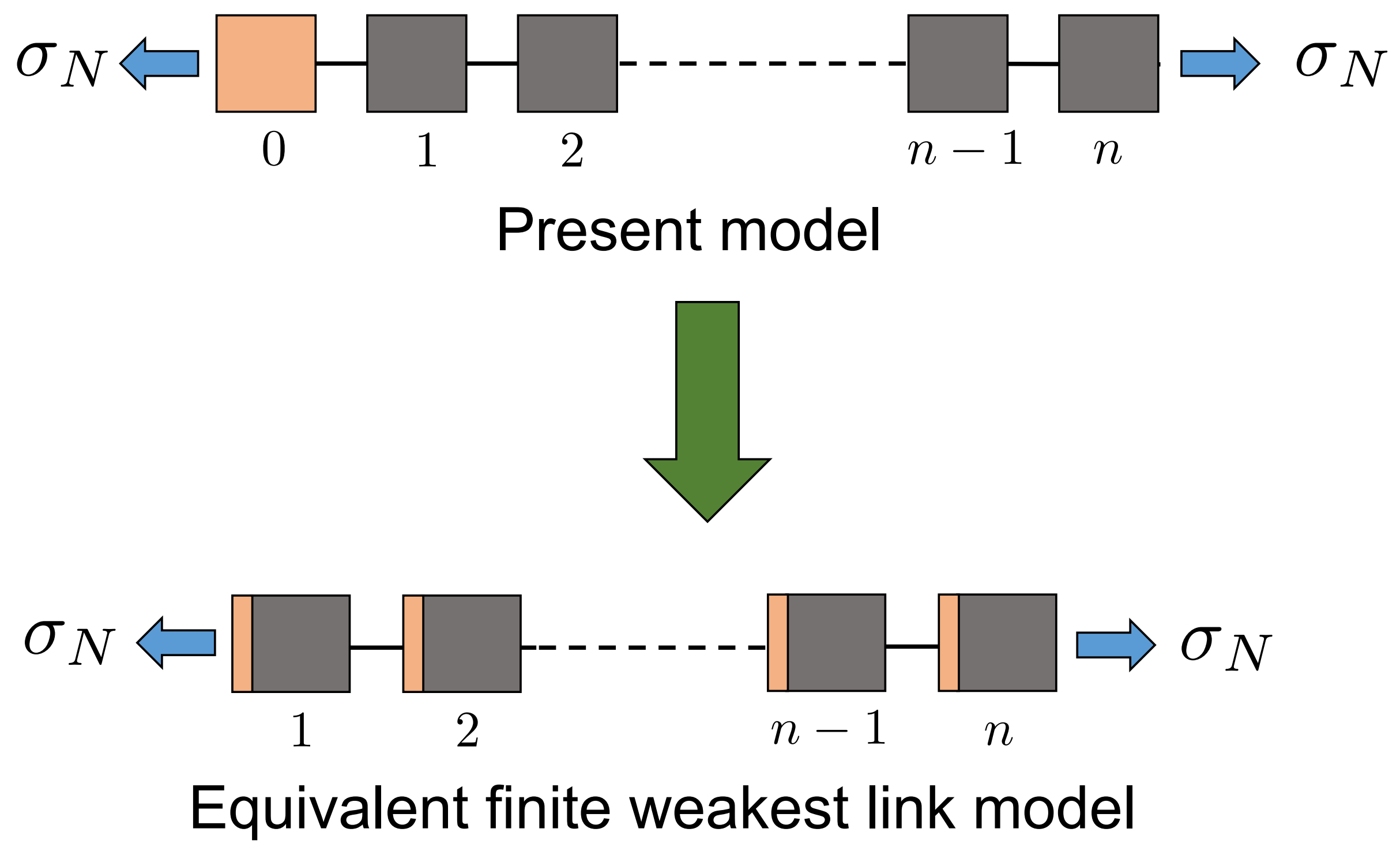

Fig. 13 